\title{
Development of Collision Resilient Drone for Flying in Cluttered Environment
}

\author{
AL MAHMUD \\ West Virginia University, am0324@mix.wvu.edu
}

Follow this and additional works at: https://researchrepository.wvu.edu/etd

Part of the Aerospace Engineering Commons

\section{Recommended Citation}

MAHMUD, AL, "Development of Collision Resilient Drone for Flying in Cluttered Environment" (2021). Graduate Theses, Dissertations, and Problem Reports. 8022.

https://researchrepository.wvu.edu/etd/8022

This Thesis is protected by copyright and/or related rights. It has been brought to you by the The Research Repository @ WVU with permission from the rights-holder(s). You are free to use this Thesis in any way that is permitted by the copyright and related rights legislation that applies to your use. For other uses you must obtain permission from the rights-holder(s) directly, unless additional rights are indicated by a Creative Commons license in the record and/ or on the work itself. This Thesis has been accepted for inclusion in WVU Graduate Theses, Dissertations, and Problem Reports collection by an authorized administrator of The Research Repository @ WVU. For more information, please contact researchrepository@mail.wvu.edu. 
Graduate Theses, Dissertations, and Problem Reports

2021

Development of Collision Resilient Drone for Flying in Cluttered Environment

AL MAHMUD

Follow this and additional works at: https://researchrepository.wvu.edu/etd

Part of the Aerospace Engineering Commons 


\title{
Development of Collision Resilient Drone for Flying in Cluttered Environment
}

\author{
Al Mahmud \\ THESIS SUBMITTED TO THE \\ Benjamin M. Statler College of Engineering and Mineral Resources \\ AT WeSt Virginia University \\ IN PARTIAL FULFILLMENT OF THE REQUIREMENTS FOR THE DEGREE OF \\ Master of SCIENCE in \\ Aerospace EngINEering
}

Guilherme A. S. Pereira, Ph.D., Chair

Jason N. Gross, Ph.D.

Nicholas Szczecinski, Ph.D.

Department of Mechanical and Aerospace Engineering

Morgantown, West Virginia

2021

Keywords: Cage, Drone, Collision, Resilience

Copyright (C) 2021 - Al Mahmud

CC BY-NC-ND 4.0 


\title{
ABSTRACT \\ Development of Collision Resilient Drone for Flying in Cluttered Environment
}

\begin{abstract}
Al Mahmud
The use of multi-rotor aerial vehicles, also known as drones, has increased significantly in the last decade due to their ease of use and applicability to an extended range of functions. Therefore, the importance of making these vehicles safe, for both themselves and the people around them, has also increased. A clear solution to this problem is the development of algorithms that generate collision-free paths using different sensors. However, even with such on-board computation, avoiding a collision is not always possible. Thus, the use of a protective structure (cage) around the aerial vehicle is a solution to keep it safe from potential accidents. A protective structure can be designed based on the application of the aerial vehicle. Different protection ideas for aerial vehicles are discussed in this work. Additionally, two protective cages have been designed, prototyped, and analyzed. The first cage has the shape of a truncated icosahedron. The drone is mounted inside the cage and is connected to it through a 3 -axis gimbal system, which prevents unwanted rotations due to collision. As a result, the drone is less likely to lose thrust and fall after any collision. The second cage is smaller than the first one since it does not contain a gimbal system. It has the shape of a turtle shell. The drone is connected to a platform in the center of the cage through a bearing, thus allowing the drone to rotate with respect to the cage in the yaw direction. Although the cage protects the drone and its propellers from any impact, it does not allow the vehicle to roll or pitch, what may cause lost of thrust after a collision. If the drone falls, the turtle shape helps the drone to reorient into its take-off position to restart its task. We present experiments with these cages and compare their performance in real flights.
\end{abstract}




\section{Contents}

1 IntRoduction 2

1.1 Problem Overview And Motivation . . . . . . . . . . . . . . . 2

1.2 Background ............................ 3

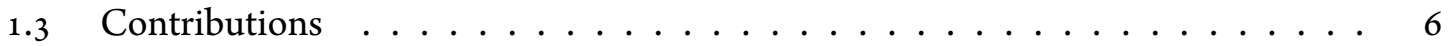

1.4 Thesis Organization $\ldots \ldots \ldots \ldots \ldots \ldots$

2 Literature Review $\quad 8$

3 Methodology 17

3.1 Drone Selection . . . . . . . . . . . . . . . . 17

3.2 Quadrotor Mathematical Model . . . . . . . . . . . . . . . . . 19

3.2.1 Basic Concepts . . . . . . . . . . . . . . . . . . 19

3.3 Truncated Icosahedron Shaped Cage . . . . . . . . . . . . . . . . . 21

3.4 Design Considerations for Truncated Icosahedron Shaped Cage . . . . . . . . 22

3.4 .13 axis Gimbal . . . . . . . . . . . . . . . 22

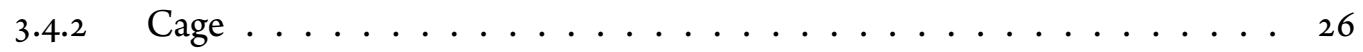

3.4.3 Concluding Remarks on the Design . . . . . . . . . . . . . 30

3.5 Turtle Shell Shaped Cage . . . . . . . . . . . . . . . . 30

3.6 Design Considerations for Turtle Shell Shaped Cage $\ldots \ldots \ldots \ldots$. . . . . 31

3.6.1 Single axis Gimbal . . . . . . . . . . . . . . . . . . 31

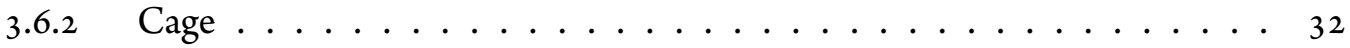

3.6.3 Concluding Remarks on the Design . . . . . . . . . . . . . 34

4 Results $\quad 36$

4.1 Experiment on the Truncated Icosahedron Shaped Caged Drone . . . . . . . . 36

4.1.1 Rolling in the Ground . . . . . . . . . . . . . . . . 37

4.1.2 Climbing Up and Down a Wall . . . . . . . . . . . . 37

4.1.3 Colliding with the Wall . . . . . . . . . . . . . 38

4.2 Flight Data Analysis on the Truncated Icosahedron Shaped Caged Drone . . . . . 39 
4 Experiment on the Turtle Shell Shaped Caged Drone . . . . . . . . . . . 40 4.3 .1 Hitting the Wall ... . . . . . . . . . . . . . . 40 4.3.2 Reorient to Take-off Position . . . . . . . . . . . . . . . . 41

4.4 Flight Data Analysis on the Turtle Shell Shaped Caged Drone . . . . . . . . . . 42

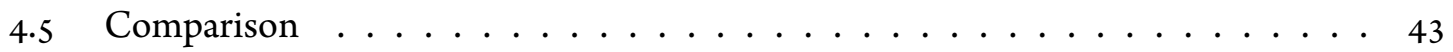

4.6 Concluding Remarks . . . . . . . . . . . . . . . 43

5 Conclusions And Future Work $\quad 45$

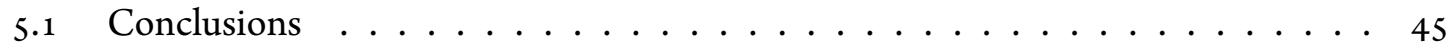

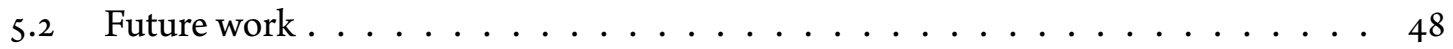

REFERENCES 


\section{List of Figures}

1.2.1 A drone is colliding with a wall in a tilted orientation. As a result frictional force is introduced in the upward direction that is causing unwanted rotation in the drone. 5

1.3.1 Contributions of this work. The figure shown on the left is the truncated icosahedronshaped caged drone. It has a 3-axes gimbal system. The figure on the right is the turtle shell-shaped caged drone. It can reorient itself to a take-off position if it is placed upside-down. . . . . . . . . . . . . . . . 6 6

2.0.1 Left: AR Drone 2.0 without any protective structure. Right: the modified AR Drone 2.0 with a protective structure, and mesh around the structure for allowing humans to touch. $[4] \ldots \ldots \ldots \ldots \ldots$

3.4.1 Three axial Gimbal mounted on the top of the drone. This gimbal is mounted in a cage, as shown in Figure 3.4.6. . . . . . . . . . . . . 23

3.4.2 A regular octagon, where a is edge length, $\mathrm{d}$ is long diagonal, and e is medium diagonal. . . . . . . . . . . . . . . . . 25

3.4.3 Left: Joint between the two edges of a octagonal gimbal ring; Right: Pivot that connects the octagonal gimbal with the cage. . . . . . . . . . . 26

3.4.4 Left: Pivot that connects the octagonal gimbal with the inner gimbal's shaft; Right: Pivot that connects with the drone. . . . . . . . . . . . . . . . 27

3.4.5 Joints of the truncated icosahedron shaped cage. Each joint has three holes, where two edges of two different hexagon is inserted, and one edge from pentagon is inserted; The joint in the left has no space holding bearing; The joints in the right has an additional hole where a bearing is mounted. . . . . . . . . . 28

3.4.6 Fully assembled truncated icosahedron shaped caged drone. . . . . . . . . . . 29

3.6.1 Single axis gimbal; (a), (b) are snapshots of gimbal position with respect to the main frame. . . . . . . . . . . . . . . . . 32

3.6.2 Left: Turtle shell shaped cage; Right: Turtle shell shaped cage with inner frame is mounted in it. . . . . . . . . . . . . . . . 33

3.6 .3 Fully assembled turtle shell shaped caged drone. . . . . . . . . . . . . . 34 
4.1.1 Snapshots when the drone is rolling on the ground. . . . . . . . . . . 37

4.1.2 Snapshot of the truncated icosahedron shaped caged drone when it is rolling along a wall. . . . . . . . . . . . . . . . . . . . 38

4.1.3 The truncated icosahedron shaped caged drone collides with a wall. In figure (a) the drone is moving towards the wall, in figure (b) the collision is happening, and in figure (c) the drone is bouncing back from the wall. . . . . . . . . . . 38

4.2.1 Changes in roll angle with time. The green curves shows the roll set by the pilot; the red curves shows the roll estimated by the drone. Y axis of the plot shows roll angles in degrees. $\mathrm{X}$ axis shows time in minutes. . . . . . . . . . . . . . . 39

4.3.1 The truncated icosahedron shaped caged drone collides with a wall. In figure (a) the drone is moving towards the wall, in figure (b) the collision is happening, and in figure (c) the drone is bouncing back from the wall. . . . . . . . . . . . . 40

4.3.2 Drone with turtle shaped cage reorient to take-off position. The reorientation progress through time in alphabetical order shown in the figure. The time step is also mentioned in the figure. . . . . . . . . . . . . . . 41

4.4.1 Flight data of turtle shell shaped caged drone obtained from pixhawk mini 4. The $\mathrm{X}$ axis represents time in minutes, and the $\mathrm{Y}$ axis represents angle in degrees. The green curves are roll angles set by the user, the red curves are roll estimated by the drone. . . . . . . . . . . . . . . . . . . . 42 


\section{Acknowledgments}

I would like to start with expressing my gratitude to my advisor Dr. Guilherme A. S. Pereira. I started my graduate studies from the point zero. On the verge of completion of Master of Science degree, I can say he was the main force behind my achievement.

I am also very grateful to Dr. Jason N. Gross and Dr. Nicholas Szczecinski for providing valuable remarks and suggestions on the course of my research work.

I want to express my sincere gratitude to my friends Bernardo Martinez, Rogerio Lima and Uthman Olawoye for their valuable suggestions during the learning process.

In the end, I would like to mention about my parents. Without their inspiration, it was impossible for me to pursue graduate studies. 


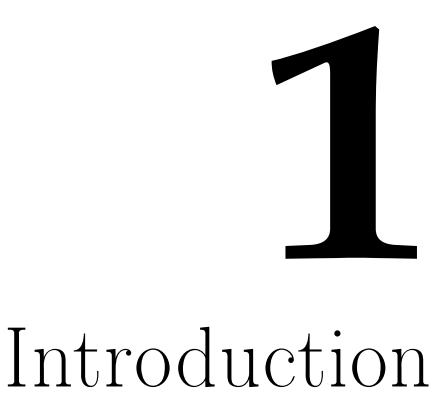

\subsection{Problem Overview And Motivation}

Unmanned aerial vehicles (UAV) are utilized for many applications including farming [32], search and salvage [21], bundle conveyance [31], investigating mines [9], and [5]. Individuals rely more upon drones for their adaptability. As the use of robots has expanded, drones need to perform more complex functions than before. As of late, drones are being utilized in observing forests [15]. A forest is a jumbled environment where trees are arranged randomly, and tree branches and leaves are generally dense. It is difficult for a drone to find a collision free way while flying inside a forest. To investigate a forest, a robot either must be extremely smart to stay away from all impacts or be sufficiently versatile to endure the crashes. A robot that can support continued collisions and proceed with capacity can effectively perform relegated undertakings in a forest-like environment. Consider an unknown, 3 $\mathrm{D}$ environment $\mathcal{W} \subset \mathbb{R}^{3}$ populated with several unknown random obstacles of arbitrary shape and size. In this thesis we assume that exploration of this environment 
is to be done with a quadrotor. The environment is unfamiliar, and there could be any number of obstacles, and they are randomly distributed. That means the distance between any two obstacles could be of any value. As the environment is in a $3 \mathrm{D}$ environment, the obstacle could be hanging from the top. There are possibilities of collision when the quadrotor is moving in the horizontal plane and vertical line. When a collision takes place, there could be many outcomes. This work aims to understand possible outcomes of a collision and develop protective structures for drones so that the drones survive strong collisions and continue the prescribed task.

\subsection{BACKGROUND}

A quadrotor is unique of UAV with four rotors that produce lift. They can be both autonomous and human-operated. By increasing and decreasing the motor's rpm, a quadrotor drone can move in all directions. It can also hover in a particular place by generating thrust equal to its weight.

The robotics environment can be both structured and unstructured. It is easier for a robot to navigate a structured environment because it has prior knowledge about the environment. But an unstructured environment is unpredictable, and the robot has to be very smart to navigate in it. As the robot has to identify changes in an unstructured continuous environment, navigating through it requires computational complexity, and sometimes collision is inevitable. In this work, the environment is considered unknown and dense, which means an unstructured environment.

An impact is an example of one moving item or individual striking fiercely against another. A crash of an aerial vehicle brings about various results. The outcomes depend on the following factors:

- The velocity of the UAV: On the off chance that the UAV's speed builds, the impact power increments. The more the crash power, the more the possibility of damage.

- The rigidity of the obstacle and elasticity of the aerial vehicle's protective structure: If the obstacle is not very rigid, the impulse force is decreased as the time of collision is increased. Similarly, if the protective structure is made of elastic material, the impulsive force can be reduced. There are many bioinspired impact-tolerant structures available, for example, hedgehoginspired structure [12]. 
- Friction with the obstacle: If both the surface of the obstacle and quadrotor are frictionless, the quadrotor will slide along the surface of the quadrotor. With the increase of relative friction between the surfaces, a frictional force is generated, which causes a rotational movement in the quadcopter.

- Direction of collision: If the direction of collision force is perpendicular to the point of contact, the frictional force will not cause rotary motion, but there will be the possibility of damage. On the other hand, if the direction of collision is angular, this angle introduces a frictional force in the plane's direction where the point of contact is presented.

If a drone collides with an object, there are several possible outcomes. First of all, consider the drone is colliding with a wall made of concrete in the direction perpendicular to the wall. Compared to a concrete wall, drone's propellers are fragile. So, if the collision force is significant, it is probable that one or more propellers can break. When a quadrotor drone loses one or more of its propellers, it encounters imbalanced thrust generation capability in different arms. To solve this issue, the control system can be improved so that a drone can balance thrust generation based on the available functional propellers. However, total thrust generation also decreases if any propeller is damaged. If the UAV frame is made of plastics, the frame will probably also break after colliding with a concrete wall. If the frame breaks, the motors' orientation will change, and in most cases, the drone crashes. A similar outcome is possible if a drone collides with a tree trunk or other rigid objects. Now consider the drone collides with a fragile object (e.g., more fragile plastic than the propeller). In this case, the object will break.

If the drone's direction is not perpendicular to the plane of collision as shown in figure 1.2.1, the frictional force acts on the drone's body towards the direction of the plane of contact. This frictional force tends to rotate the drone's body. If the frictional force is large enough to rotate the drone to a significant angle, the thrust force in the upwards direction will decrease, and, as a result, the drone will lose altitude and eventually fall.

To prevent these possible outcomes, several research works have been performed. One of the most effective solutions is the development of an obstacle avoidance algorithm. Using this technique, a drone uses different sensors to identify obstacles present in its environment. However, due to these algorithms' computational complexity and inability to detect an obstacle in different 

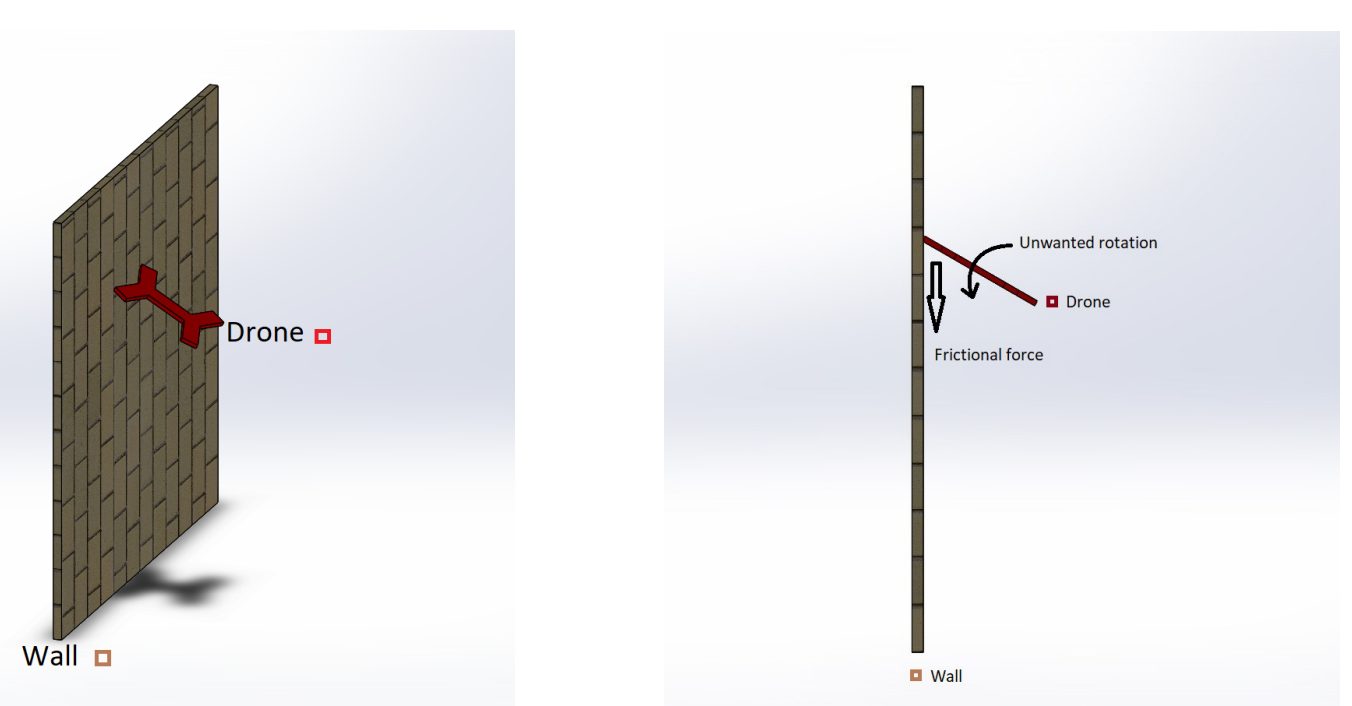

Figure 1.2.1: A drone is colliding with a wall in a tilted orientation. As a result frictional force is introduced in the upward direction that is causing unwanted rotation in the drone.

situations, we cannot rely entirely on obstacle-avoiding techniques.

To make a drone's flight safe for both itself and the environment around it, protective structures have been developed. Some protective structures guard the propellers so that after a collision, they do not break. Some propeller guards keep the propellers secured from possible collision from all possible directions [4]. On the other hand, some protective structures guard the whole drone so that the propellers and the electronic components also stay safe [7]. This type of protection reduces collision forces and lessens the probability of breaking important parts of the drone. But due to collision, frictional force exerts on the drone's protective structure, which eventually acts on the drone's body, causing the unwanted drone rotation. These unwanted rotations destabilize a drone's natural flight and may cause a crash as well. To mitigate the effect of frictional force, control strategies help identify disturbances due to a collision and generate proper command to overcome the disturbances. There are other strategies available to mitigate the effect of frictional forces. This includes giving the protective structure the ability to rotate with respect to the drone. Thus, following a collision, the protective structure will rotate due to the frictional force, which will not affect the drone's body. In most cases, a protective structure has a rotation ability about the yaw axis, which does not help much to mitigate the possibility of unwanted roll and pitch after a collision. Some works use a 3 -axes gimbal system so that all the unwanted rotation can be minimized [7]. 
The development and fabrication of two protective structures are the main contributions of this work, as presented next.

\subsection{CONTRIBUtions}

In this work, a 3-axes gimbal-based protective structure was developed. The effectiveness of this type of protective structure are evaluated, and the advantages and disadvantages are discussed.
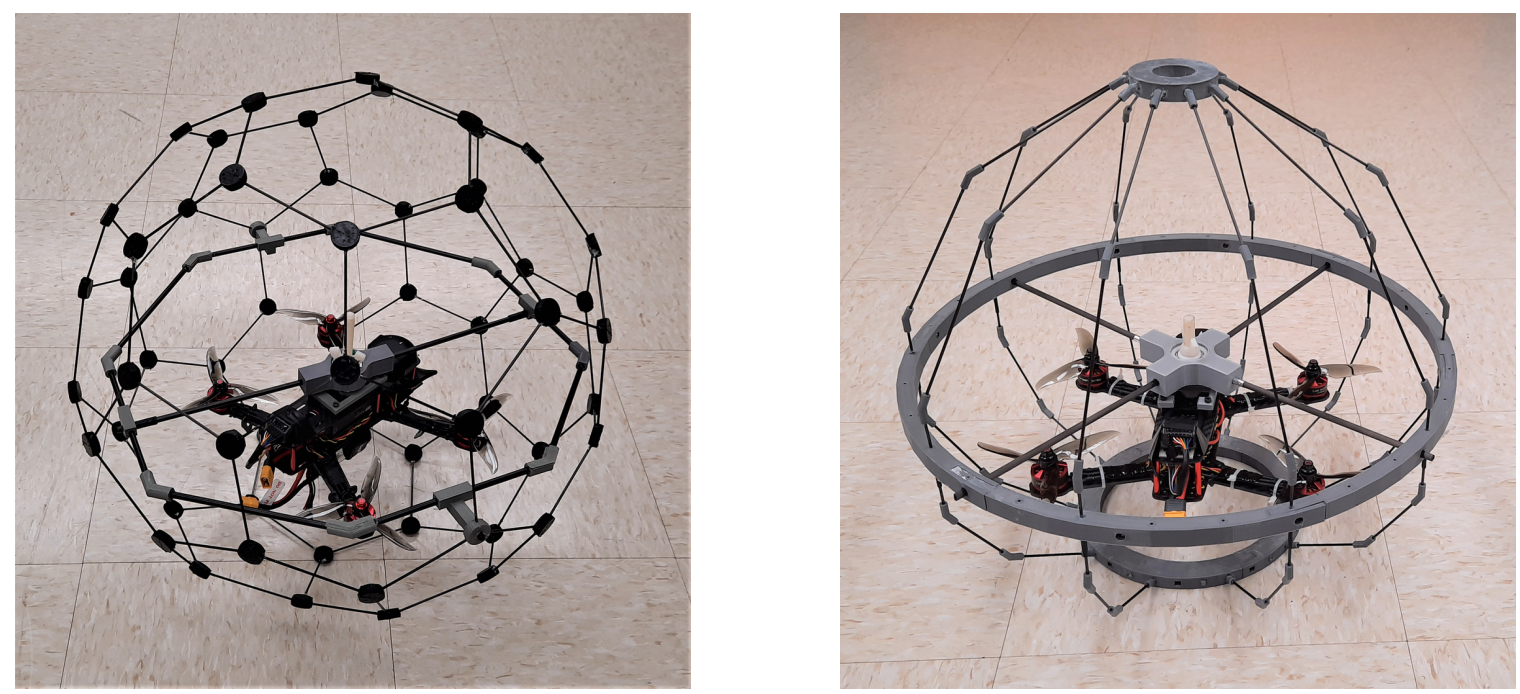

Figure 1.3.1: Contributions of this work. The figure shown on the left is the truncated icosahedronshaped caged drone. It has a 3 -axes gimbal system. The figure on the right is the turtle shell-shaped caged drone. It can reorient itself to a take-off position if it is placed upside-down.

After discussing a 3-axis gimbal-based caged quadrotor, another proposal for a protective structure for quadrotors will be presented. The proposed protective structure does not have a 3-axis gimbal system, so it is likely to fall after a significant collision. However, this structure has been inspired by the working principle of turtle shells, where a shell helps a turtle reorient into a natural position after getting upside-down [1 1]. So, after a collision, if this caged drone crashes into the ground, it will roll back to a stable position so that the drone can take off again. In figure 1.3.1, both protective structures are shown. 


\subsection{Thesis Organization}

This work aims to develop two collision resilient caged drones and analyze the effectiveness of the drones based on several experimental observation and flight log analysis. The parts of the models are designed using Solidworks-2020 and printed using Ultimaker Cura 3. The thesis report contains five chapters. The organization of the thesis is discussed as follows.

- Chapter 1, Introduction: In this chapter, the general idea of the problem has been discussed. After that, the available solution for the discussed problem and the solution's scope has been presented. Finally, the contribution of the work has been summarized.

- Chapter 2, Literature Review: Previously, several research works were performed to find a solution to the problem stated in chapter 1 . In this chapter, a brief overview of the previous results has been summarized.

- Chapter 3, Methodology: Based on the knowledge obtained by studying earlier research works, two caged drones have been designed and built. The process of manufacturing the two models, the constraints in the manufacturing process, and the modification of designs due to the constraints have been discussed in this chapter.

- Chapter 4, Results: In this chapter, the two caged drones' experimental results have been discussed. After experiments, the flight data have been analyzed, comments on the two designs have been made.

- Chapter 5, Conclusion: In this chapter, the work outcomes have been summarized. Based on the findings, a recommendation for future works has been made. 


\section{2 Literature Review}

An autonomous robot has to visualize the unknown environment to function correctly. A drone can build a complete map of its surroundings by using different sensors. With the development of electronics and sensors, numerous research has carried out to detect and avoid obstacles. Many obstacle detection and avoidance techniques are suitable to implement in aerial vehicles. As different aerial vehicles perform different tasks, choosing an appropriate approach to detect and avoid obstacles depends on the drone's configuration and its assigned job. Previously distance sensors [26], stereo vision sensor [22], laser scanner [14], and many more sensors have been used successfully to detect and avoid obstacles for enabling a drone autonomous flight. However, despite developing great algorithms and using developed sensors, these works cannot guarantee obstacle avoidance in all platforms. In most cases, the obstacles are big walls or humans or boxes, etc. But, in real-world applications, obstacles may be much more complex. When the environment is very cluttered and dense, or the obstacles are too difficult to detect, or the visual condition is poor, collision with the obstacle is, in most cases, unavoidable. So, for aerial vehicles with restricted movement and dif- 
ferent sizes, collision and sometimes crash is inevitable. There are several approaches to generate a complete map of the surroundings of a flying robot. A robot can operate autonomously in an unknown environment by applying obstacle avoidance techniques in a $3 \mathrm{~d}$ map and using $3 \mathrm{~d}$ path planning. However, making a complete map and planning a path while avoiding obstacles needs much calculation, which requires high power multicore CPU systems. Carrying a high-power CPU increases weight, and so the thrust generation needs to be greater. So, more powerful motors and propellers are required. So, overall power consumption increases, and flight time reduces. When the focus is to minimize the weight and maximize the flight time, an off-board CPU is preferable. But this requires communication over a wireless link. This wireless communication has some waiting time between obstacle detection and avoidance. If the waiting time increases, a drone is more likely to collide with an obstacle. As the latency increases, collision is inevitable in most cases. So, low power optical sensors are used to avoid obstacles [27], [6]. But, optical flows sometimes have problematic estimation [25]. Considering the above situations, researchers have started to emphasize developing protection mechanisms for drones. Based on the drone's configuration, functionality, and application, different techniques to protect a drone have been carried out.
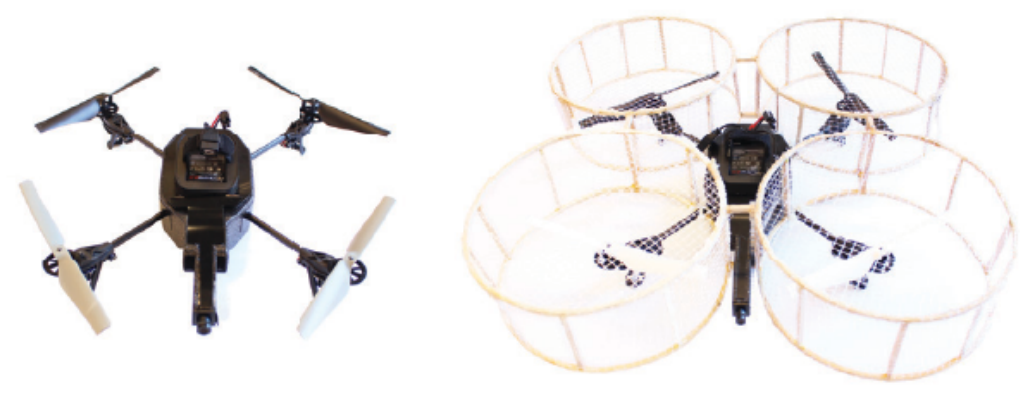

Figure 2.0.1: Left: AR Drone 2.0 without any protective structure. Right: the modified AR Drone 2.0 with a protective structure, and mesh around the structure for allowing humans to touch. [4]

A protective structure performs two goals; it protects the drone's components from breaking due to collision, and it protects its surroundings from damage by the drone's propellers. As the propellers are most prone to break after an impact and cause damage to the object they collide with, the basic technique is to design a protective structure around each of the propellers. In figure 2.0.1, a similar idea has been implemented [4]. In this work, a light-weight wooden frame surrounds 
each of the propellers. The frame's shape is right circular cylindrical. Two wooden rings form the top and bottom disk, connected with eight wooden sticks to make the cylindrical structure. A clear polypropylene mesh covers the surface area. This mesh prevents direct contact with the propellers. So, it is safe both for the propellers, as well as the surroundings.

Collision induces a force on the drone. Making a protective structure can eliminate the possibility of damage to the components. To mitigate the possibility of change in the drone's attitude after a collision, developing a control strategy is a solution. An excellent example of developing a collision recovery control system is presented in [10]. The idea is to use a quadcopter with the propellers protected and apply a recovery control upon collision. When a collision with a vertical wall occurs, the collision force may change the drone's orientation that eventually can disturb the drone's stability. The recovery control system regains the drone's stability and drives the drone in a safe flying condition. The result shows that the recovery control system is successful for collision speed up to $2 \mathrm{~m} / \mathrm{s}$ and inclination of $17^{\circ}$. Nevertheless, this control system requires extensive calculation and prompt response. As a result, despite having a protective structure, the need for high power CPU is essential in this case. In this design, around each of the propellers, ${ }_{3} \mathrm{D}$ printed nylon bumper is placed. Two carbon fiber tubes connect a bumper with the adjacent bumpers. The bumper protects the drone from a vertical collision. Following a collision, the impact is measured, and the value is returned to the controller. The controller uses this information to generate control forces to stabilize the drone.

The designs discussed so far focus on securing propellers from their surroundings. Some researchers focus on protecting the sensitive equipment of the drone [8]. The platform of a drone contains all the necessary electronics. If any electronic components fail, the flight becomes unsafe. That is why securing the electronic components is a notable concern.

Up until this point, we have discussed about protective structures produced using materials with rigid materials. These materials cannot reduce collision forces [30]. So, the structures need to be very strong and heavy. To make lightweight, protective structures researchers recommends origami-inspired design [30]. Their suggested origami structures absorb energy and reduce collision energy. They further added that using a rotary protective structure is better than a fixed protective structure. The protective structure can turn about the robot's yaw pivot. For both oblique and normal collision, the collision force decreases when origami-protected structure is used instead of naked protective structure because the collision duration is significantly longer for origami- 
protected structures. It is further observed that, a rotary protective structure and a fixed protective structure behave similarly when the force is normal. But, when the force is oblique, the collision force reduces when rotary protective structure is used. Moreover, angular speed is much higher for the fixed protective structures than the rotary protective structures.

Some researchers prefer a protective structure that can absorb collision energy. There are many ways to make the protective structure absorb collision energy. One such way is making a protective structure using Euler Spring [16]. An Euler spring is a narrow column that is bent and loaded axially and kept in a balanced state [19]. So, when any force is applied to the two sides of the column, the column bends to compensate for the pressure. In this design, a tetrahedral structure has been developed. The edges of this structure are made of Euler springs. Whenever any collision occurs, the edges buckles, so the frame also buckles, and thus the impact energy is absorbed. The amount of energy that can be absorbed depends on the length of the springs. If the length is increased, more energy is supposed to be absorbed, but the size of the frame will increase, resulting in a bigger size of the robot. The design is very good for compensating impact of a large amount of force. The weight of the frame is not too much, which is an advantage, but the frame increases the size of the robot. So, if the environment is dense, this configuration will not be suitable. This design is best suited if the collision is expected to be with concrete or any surface with a larger area.

Work has been done to protect the drone from a collision in [23]. This idea is inspired by the wings of the wasp in which a flexible, resilient joint is present. This design permits the wingtip to flex marginally during the flight, which is the firm state, and let it fold along a bend line when any collision happens. They adopted this strategy for the protection of the frame of multirotor aerial vehicles. Their proposed approach depends on two components that work in collaboration. The first component is a frame with dual-stiffness properties, which helps transit reversibly between inflexible and flexible states to achieve mechanical compliance. The second component is a case guarded by energy-retaining materials, which permits ingesting an effect's energy and saving the body. The proposed strategy is good when considering collision in the lateral axis. But, they have not mentioned any solution to mitigate a collision in the vertical axis. Moreover, the propellers are designed to stay protection-free. If any branch of a tree enters into the area of the propeller, it can break. They have suggested that individual cages around the propeller can be designed to protect the propeller. If that is done, the overall weight will increase. They have not mentioned whether the current motor and battery configuration will be able to carry that added weight. The total weight 
at the current configuration is 51 grams. If the weight is increased 4 or 5 times, the momentum will increase for the same speed. So, the impact of collision will be higher. This strategy does not provide any insight into how to fix a multirotor vehicle with a larger weight.

In [7] a model, named GimBall, has been designed and developed. The idea is to make a protective structure around the inner frame to keep the inner frame secured from collisions. This protective structure is built to absorb impact energy without cracking, and it ought to be adequately solid to secure the inner parts. The gimbal system is used to compensate for the disturbance after any collision so that the inner frame stays steady and stabilized. The inner frame is designed to hold all avionics to allow autonomous flight. All the sensors and control electronics are mounted in the inner frame in a way that the center of mass is adjusted with the axes of rotation to ensure proper mitigation of the disturbances. The design idea is good, but there are some issues to be taken care of. First of all, the design is based on a twin coaxial rotor system. This type of system is very complex. If torque imbalance occurs, it will make the yaw uncommanded. The two rotors will rotate in the opposite direction. So, the upper rotor will feel a downward force due to lift, whereas the lower rotor will feel an upward force. So, there is a possibility for the rotors to be intersected. The study mentions this design is suitable for protecting the drone when moving in the forest. But the outer frame can protect the gimbal and inner frame by absorbing energy when it collides with something like a wall or stems; small branches can still enter through the open spaces in the outer frame and disturb the inner frame as can stop the rotation of the propeller. The gimbal compensates and keeps the drone in the same direction of motion. So if any branch enters through the outer frame from the top and the drone is also moving towards up, the drone will go down slightly after any collision. Because of the gimbal system, it will again move towards up. As a result, the drone could be stuck in a loop of going up and down.

A study designed and fabricated a protective structure with a radius of variable curvature [24]. The material used for this design is carbon fiber. The variable curvature helps to minimize negative aerodynamic effects. Using carbon fiber makes the protective structure strong while keeping the weight light. This proposal does not mention the drone configuration. So, it is not specified how much lift needs to be generated to work this structure.

Two hemispherical rotating shell is proposed as the protective shell [28]. These two hemispherical shell can rotate independently. The purpose of the gap between the two hemispherical shell is to allow physical interaction and power tathering. For physical interaction, manipulation system can 
be positioned along the roll axis which lies between the gap of the two hemispherical shell. The proposed system is good as it considers possible physical interaction and power tathering. But, as the two hemispherical shells rotate independently, there could be estimation errors in rotation changes after a collision due to different rotation rates. Moreover, this system does not allow yaw rotation because of the presence of the manipulation system. The manipulation system is designed to put in the roll axis, which is supposed to move in response to any collision. This will move the end-effector in a disorganized way which is not desired.

In order to protect the propellers a design has been proposed that includes rotating hemispherical shells around every propeller [29]. This design will provide protection and collision resiliency. As, the protection can be developed around every propeller, this design allows different type of configuration like trirotor, quadrotor etc. The motivation behind this design is to protect the propellers as well as to keep the camera view clear. Because, if the shell rotates around the UAV body, it sometimes blocks camera view, which results in unwanted behaviour. As the rotating shells will be around the propellers only and the camera will be mounted at the top or bottom of the UAV body, the camera view will always stay clear. The design solves the issue with the camera view very well. But, two rotating shell around each propeller will make the drone very heavy. As per the requirements of a mission every drone requires some equipments to carry with it. So it is necessary that the overall weight is kept as low as possible. Two rotating shell for every propeller introduces total eight rotating shells which will add too much weight.

A foldable cage is developed [17] to minimize the storage space. The cage is designed to protect the drone and the parcel carried by the drone. The cage can be folded when it is not being used and needs to be stored. The idea of a foldable cage is good. But to make a cage foldable, the cage has to be very big compared to the drone's size. So the overall size of the system increases. If the size increases, the accessibility of the drone in the dense area will reduce.

Another drone intended to be implemented in parcel services has been developed by making a cage around the drone [18]. The cage is designed for two reason: first, to protect the parcel, carried by the drone and second to protect humans from getting hurt by the propellers. Its propeller can retract during take off and landing but extends while flying which helps increase efficiency and stability. If during flight the propeller stays outside, they could break if collide with the environment. But, the idea of retracting propeller is good because, in that case, we can add more equipment with the drone but the airflow will not get obstructed. We can modify this design by keeping the 
propeller guarded even when it is extended.

There are some different kinds of the protective system as well. One of these protective system is the icosahedron tensegrity structure that has been developed to protect the drone [34]. The frame is made of rigid bodies. It is designed to distribute the load among the members, and the rods do not undergo bending moments in impacts. The structure is very complex. If the size of the drone increases, the complexity increases.

Some drones can change orientations to fit in different pathways [13]. Like a bird, this drone can squeeze and penetrate through small gaps. This design is good, considering the drone can access through gaps of different shapes by squeezing the body. But, this change in form changes the center of gravity which makes the control very complex. Moreover, the design is more susceptible to instability after any collision.

A multirotor configuration is developed that can change its orientation to grab an object and move it to a different place [35]. This design is good, although the control strategy is very complex as the center of gravity changes with orientation change. This design can be modified to do another task like measuring a cylinder.

Table 2.0.1 summarizes the studies that have been discussed in this chapter. We can observe from the table that carbon fiber materials are most preferred for constructing protective structures because carbon fibers are lightweight and strong. Keeping the propellers safe from collision is one of the most important purpose of a protective design. That is why preventing collision along the lateral axis is given more importance. But, protecting the full body of the drone is preferable. The energy-absorbing capability of the protective structure is another vital consideration to reduce damage due to collision. To allow the protective structure to absorb energy, different structures have been made, and different materials have been used. Rotary protective cages are preferred to minimize collision forces. In this regard, the priority is to make a protective structure able to rotate about all three axes. If it is not possible to allow rotation about three axes, it is better to allow rotation about at least one axes than making a fixed protective structure. For some designs, it was not possible to allow rotation; in those cases, a control strategy was developed to prevent the drone from falling into the ground. If a control strategy is not used to avoid rotation, the protective structure that can reorient to a normal position after falling into the ground can be developed. The protective structures are made of small parts instead of using large parts to increase stiffness. 
Table 2.0.1: A comparison among different protective techniques.

\begin{tabular}{|c|c|c|}
\hline Name & Configuration & Specification \\
\hline $\begin{array}{l}\text { Safe-to-touch } \\
\text { Drone }\end{array}$ & Quadrotor & $\begin{array}{l}\text { Protection around propellers, use of } \\
\text { wood and mesh, cannot rotate }\end{array}$ \\
\hline $\begin{array}{l}\text { Recovery Control } \\
\text { Strategy }\end{array}$ & Quadrotor & $\begin{array}{l}\text { Fixed protection, protection for hori- } \\
\text { zontal collision, presence of recovery } \\
\text { control systems }\end{array}$ \\
\hline AR Drone & Quadrotor & $\begin{array}{l}\text { Unprotected propellers, electronics } \\
\text { are guarded, fixed protection }\end{array}$ \\
\hline $\begin{array}{l}\text { Miura-O-Ring } \\
\text { Structure }\end{array}$ & Quadrotor & $\begin{array}{l}\text { Protection for horizontal collision, ro- } \\
\text { tary protective structure, energy ab- } \\
\text { sorbance capability using origami }\end{array}$ \\
\hline $\begin{array}{l}\text { Euler Spring Pro- } \\
\text { tection }\end{array}$ & Quadrotor & $\begin{array}{l}\text { Energy absorbance capability, cannot } \\
\text { rotate, can reorient if falls into ground }\end{array}$ \\
\hline Gimball & $\begin{array}{l}\text { Coaxial } \\
\text { rotor }\end{array}$ & $\begin{array}{l}\text { Rotate around 3-axes, protects full } \\
\text { drone, use of carbon fiber rods }\end{array}$ \\
\hline Pico & Quadrotor & $\begin{array}{l}\text { Carbon fiber structure, cannot rotate, } \\
\text { cannot reorient if falls, protect drone } \\
\text { from all direction }\end{array}$ \\
\hline $\begin{array}{l}\text { Two hemisherical } \\
\text { shell (drone) }\end{array}$ & Quadrotor & $\begin{array}{l}\text { Rotate about 2-axes, cannot rotate } \\
\text { about yaw axis, protection from all di- } \\
\text { rection, carbon fiber rods }\end{array}$ \\
\hline $\begin{array}{l}\text { Two hemisherical } \\
\text { shell (each rotor) }\end{array}$ & Quadrotor & $\begin{array}{l}\text { Protects propellers only, cannot rotate, } \\
\text { cannot reorient if falls, use of carbon } \\
\text { reinforced material }\end{array}$ \\
\hline Foldable cage & Quadrotor & $\begin{array}{l}\text { Cannot rotate, cannot reorient if falls, } \\
\text { propeller protection only, use of car- } \\
\text { bon fiber beam }\end{array}$ \\
\hline $\begin{array}{l}\text { Tensigrity struc- } \\
\text { ture }\end{array}$ & Quadrotor & $\begin{array}{l}\text { Cannot rotate, can reorient if falls, full } \\
\text { body protection, use of carbon fiber } \\
\text { rods }\end{array}$ \\
\hline Squeeze and fly & Quadrotor & $\begin{array}{l}\text { Can squeeze to avoid obstacles, no } \\
\text { physical protective structure }\end{array}$ \\
\hline $\begin{array}{l}\text { Multirotor manip- } \\
\text { ulation }\end{array}$ & Quadrotor & $\begin{array}{l}\text { Multirotor changes orientation, pro- } \\
\text { tection around propellers, can avoid } \\
\text { obstacles by changing orientation }\end{array}$ \\
\hline
\end{tabular}


These are the major observations from this investigation. Based on the information obtained from this study, two protective structures have been prototyped. In the next chapter, we will discuss the design consideration and manufacturing process of the prototyped protective structures. 


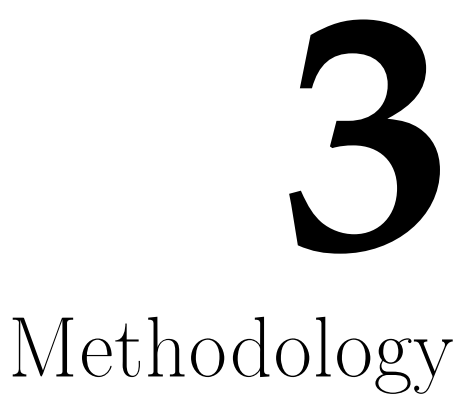

In the previous chapter, we have discussed different approaches to protect a drone. We have compared the design strategies and found out the major considerations. Based on the findings from studies, two protective structures have been developed. In this chapter, the design considerations, strategies, developed work, and their limitations will be discussed.

\subsection{Drone Selection}

The selection of a suitable drone was the first consideration in the design process. While many researchers prefer hexacopters for having more control input some others prefer single rotor drones with additional control unit. After studying several research works, a quadrotor drone was selected for this project because quadcopter-dynamics are comparatively straightforward while quadcopters are easy to build, and compact.

According to the design considerations, the QAV250 kit has been chosen. This kit includes the 
components in Table 3.1.1.

Table 3.1.1: Components of QAV250 Kit.

\begin{tabular}{||cc||}
\hline Component Name & Description \\
\hline \hline Frame & Carbon Fiber Airframe \\
\hline Motors & DR2205 KV2300 \\
\hline Propellers & Cyclone 5040c \\
\hline Autopilot & Pixhawk Mini 4 \\
\hline GPS & Pixhawk4 GPS \\
\hline ESCs & BLHeli S ESC 20A \\
\hline Telemetry Radio & $433 \mathrm{MHz}$ \\
\hline
\end{tabular}

The frame of the drone is made of carbon fiber. The mid plane is mounted on the bottom plane. Between these two frames, the arms are mounted. The flight controller plate is mounted above the mid plane. The pixhawk 4 mini is mounted at the top of this plate. When all the electronics connections are installed, the top plane is mounted with the mid plane using frame hardware. There are four motors available in the $\mathrm{QAV}_{25}$ o kit. Two of them rotate clockwise; the other two rotate counterclockwise. The motors are mounted in the arms of the carbon fiber frame. There are particular spots in the arms reserved, especially for motors. The propellers are made of plastic. They are mounted at the top of the motors using nut. The function of pixhawk 4 mini controller is to direct the motors RPM based on the input command. It also uses sensors to determine vehicles' states. The pixhawk 4 GPS helps acquire position when flying the drone outside. The QAV250 power distribution board comes with fully assembled electronic speed controls(ESCs). The function of power distribution board(PDB) is to distribute power from the flight battery to all different components of the quadrotor. The telemetry is a computerized two-way information stream, which can both send information about the trip down to a ground station (for our situation, the Mission Planner) and send order up to the autopilot.

The DR2205 KV2300 motor can generate up to 1100 thrust when cyclone 50400 propeller is used. The four motors together generate a thrust of 4400 . In general, the entire payload of a drone, including the drone's weight, should be less than half the total generated thrust. So, the maximum allowed weight, including payload, for the selected configuration is $2200 \mathrm{~g}$. The drone 
kit's weight is $350 \mathrm{~g}$. To power the system, $1300 \mathrm{mAh} 14.8 \mathrm{~V}{ }_{95} \mathrm{C}{ }_{4} \mathrm{~S}$ LiPo battery has been used, weighing $150 \mathrm{~g}$. So, the total weight of the drone is $500 \mathrm{~g}$. As the total allowed weight is $2200 \mathrm{~g}$, the payload's maximum weight could be 1700 . If we consider developing a protective structure of about 300 , the drone can still carry a load of 1400 .

\subsection{Quadrotor Mathematical Model}

Before starting the design considerations, we need to discuss some basics related to quadcopters. Consider an unknown, ${ }_{3} \mathrm{D}$ environment $\mathcal{W} \subset \mathbb{R}^{3}$ populated with many unknown random obstacles of arbitrary shape and size. A drone has to perform a task in that environment. The drone can search for a safe path using obstacle avoidance algorithms while performing an assigned task. However, consider the environment be so cluttered that it is not possible to avoid obstacles. In that case, the drone has to either stop moving or fly, finding a less risky path. If the drone continues moving, a collision is probable to happen. When a drone collides with an obstacle, there are several plausible outcomes. Besides breaking the propellers, the drone can become unstable. Because when any propeller fails, the thrust generation reduces, and so, thrust generation of different motor differs. In the worst possible case, the drone can fall. In the next section, fundamental concepts related to quadrotor drones will be discussed.

\subsubsection{BASIC CONCEPTS}

In the quadrotor, there are four motors structured in a cross $(\mathrm{X}$ or +$)$ configuration. Each rotor of a motor is connected to a propeller. The motors are mounted so that the motors' rotor mounted in the same arm rotates in the same direction. The rotors of the motors mounted in the other arm rotate in the opposite direction. That means the rotors of the two side-by-side motors rotate in opposite directions. A quadrotor has $6 \mathrm{DOF}$; those are the three coordinates $\mathrm{X}, \mathrm{Y}, \mathrm{Z}$, and the three attitude angles $\theta, \varphi, \psi$. Notice that it has only four rotors that control this $6 \mathrm{DOF}$ system. That means the quadrotor is underactuated system and four control inputs can be chosen using the four rotors to get the desired attitude.

- Throttle, $\mathrm{T}$, is obtained by controlling the speed of the propellers. This force acts vertically. By increasing the force we increase the height of the quadrotor and by decreasing it we make 
the quadrotor to lose altitude. If the quadrotor is upside down with no rotational force, increasing thrust causes the drone to fall as thrust acts downward in this case. All the motors should generate the same amount of force for obtaining linear movement vertically. If all the motors generate maximum force, then thrust will be full (considering all the motors are equally efficient).

- Roll, $\theta$, is a rotational movement about one of the horizontal axes. Considering the motor generating force $\mathrm{F}_{1}$ is mounted along the $\mathrm{X}$-axis, the roll is obtained by increasing or decreasing force generated by any one of the motors between $\mathrm{F}_{2}$ and $\mathrm{F}_{4}$. If the right propeller's speed is increased, the roll will be counterclockwise, and if the speed of the left propeller is increased, the roll will be clockwise.

- Pitch, $\varphi$, is the rotational movement along the other horizontal axis. This time, the pitch is obtained by increasing or decreasing one of the propellers' speed between $F_{1}$ and $F_{2}$. If the front propeller's speed is increased, the pitch will be counterclockwise, and if the speed of the back propeller is increased, the pitch will be clockwise.

- Yaw, $\psi$, is the rotational movement along the vertical axis. It is obtained by increasing or decreasing the couple of front/rear propellers or other propellers. If the front/rear couple of propellers are rotating clockwise, increasing their speed will result in yaw in the clockwise direction and vice versa.

The accelerations along three axes are obtained from the following equation [20].

$$
\left[\begin{array}{c}
\ddot{x} \\
\ddot{y} \\
\ddot{z}
\end{array}\right]=F_{z q} / m\left[\begin{array}{c}
\cos \psi \sin \theta \cos \varphi+\sin \varphi \sin \psi \\
\sin \psi \sin \theta \cos \varphi-\sin \varphi \cos \psi \\
\cos \theta \cos \varphi
\end{array}\right]-1 / m\left[\begin{array}{c}
f_{x} \\
f_{y} \\
G+f_{z}
\end{array}\right]
$$

Here, $F_{z} q$ is the force along $\mathrm{Z}$ axis, $\mathrm{m}$ is the mass of the drone, and $f_{x} f_{y} f_{z}$ are drag forces, $\mathrm{G}$ is the force due to gravitational acceleration. From equation (3.1), we can observe that upward direction acceleration depends on the roll and pitch angles. Due to a collision, if the frictional forces introduce rotation in the drone's body, the roll and pitch angles will increase. If the roll and pitch angles increases, their cosine value decreases, which eventually reduces the acceleration towards positive 
$\mathrm{Z}$ direction according to equation (3.1). As a result, the drone experiencing rotation, will lose altitude, and eventually fall into the ground. This phenomenon motivates us to develop a protective structure that mitigates changes in roll and pitch angle after a collision happens.

\subsection{Truncated Icosahedron Shaped Cage}

There are many protection mechanisms to protect the drone's components. Some protective structures are meant to protect the propellers [4], [10], [29], some protective structures protects the electronics [8], some other protects the entire drone [16], [7], [24], [28], [28], [34]. Many researchers consider circular protection around a drone [30]; thus, when a horizontal collision occurs, the protection mitigates the impact. As there is no protection for vertical collision, this protective structure is not suitable for applications when a drone needs to move up and down repeatedly. This kind of protective structure holds no good when a vertical collision occurs. Some researchers focus on developing a control strategy to control the drone's attitude following a collision [10]. Some other prefers using rotary protective structure to minimize the change in attitude due to collision [30], [7].

A protective cage is ideal if it can provide protection all around an object. If any side of a drone is open to collision, the drone is not secured. For example, if there is a circular protective structure like [30], there is no protection if there is a collision from the drone's top or bottom. A drone moves in 3-dimensional space. So, a collision can be horizontal, vertical as well as oblique. That is why protecting a drone from a horizontal collision is not sufficient. Moreover, the mainframe of a drone contains critical electronics and control systems. If this part breaks due to collision, there is no use of the fully secured propellers. That is why there are protective mechanisms available to protect only the mainframe [8]. After studying different protective mechanisms, we choose to develop a structure that can protect the drone from vertical, horizontal, and oblique collision. The previous section discussed that increasing roll and/or pitch angles cause a reduction of acceleration in the positive $\mathrm{Z}$-axis. If a protective structure can rotate with respect to the drone due to a frictional force, the attitude of the drone should not change. That is why we want the protective structure to rotate around the drone. Such a cage could be a rectangular-shaped protective structure because it protects from all directional collisions. Nevertheless, a rectangular cage has sharp corners and does not maintain uniform and smooth contact with the surface. Moreover, a rectangular cage 
only allows rotation around the yaw axis unless the cage's height equals the width of the cage.

A circular protective structure can maintain uniform and smooth contact with the surface if the circular structure moves perpendicular to the direction of the surface. For this reason, most of the protective structures are made circular $[4],[30],[24]$. A spherical protective structure can always maintain smooth contact with the surface because it always moves perpendicular to the collision surface [7], [28], [29]. However, a spherical protective structure can be improvised and made more efficient. As protective frames cannot be made perfectly smooth to reduce frictional force due to collision, a gimbal system can be used. As the gimbal system allows the protective structure to rotate with respect to the drone, collision forces do not affect in drone's attitude change, thus allowing the drone a safer flight. The gimbal system may also have friction and may not rotate smoothly. Bearings can be used in the pivots of the gimbal system to enable frictionless and smooth rotation of the gimbals.

\subsection{Design Considerations for Truncated Icosahedron Shaped Cage}

collision can possibly break the parts of the drone and introduce rotational movement in the drone. It is necessary to prevent damage as well as rotation after a collision. This section will discuss the design and manufacturing process of the truncated icosahedron shaped caged drone. The design focuses on solving both the problem simultaneously.

The design consists of the following parts:

- Three axis Gimbal allows the drone to rotate freely with respect to the cage and the cage itself, with an almost circular shape.

- The cage protects the drone, mounted inside it from collisions from all directions. It can rotate around the drone to mitigate unwanted roll and pitch of the drone.

\subsubsection{AXIS GIMBAL}

A gimbal permits an item to turn about an axis. A gimbal, mounted symmetrical to another gimbal, permits turning along two different axes. In this work, three gimbals have been mounted orthogonal to one another to enable the robot to rotate about three axes. For smooth and frictionless rotation, a bearing is used in the pivot. As shown in the figure 3.4.1, the connection at pivot 1 enables 
the drone to rotate about the Z-axis, pivot 2 and 3 allows rotation about Y-axis, and pivot 4 and 5 connected to the cage allows rotation in $\mathrm{X}$-axis. The gimbal structure was constructed using carbon fiber rods, and 3-D printed joints, ensuring modularity as the rods are connected at the joints and can be easily replaced. The gimbal was carefully assembled so that all the pairs of rotational pivots are aligned with each other and centered about the middle of the cage.

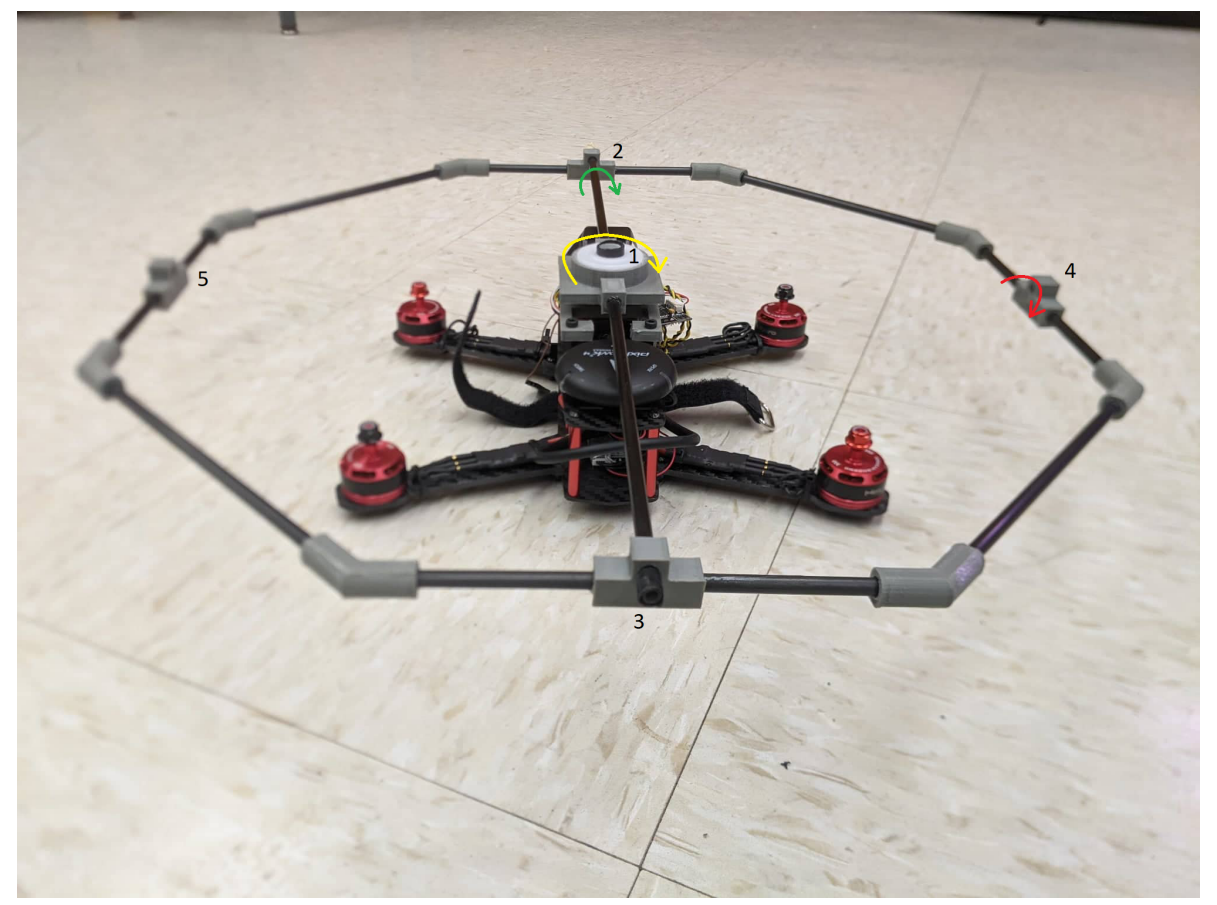

Figure 3.4.1: Three axial Gimbal mounted on the top of the drone. This gimbal is mounted in a cage, as shown in Figure 3.4.6.

The design considerations of the gimbal is as follows:

- The gimbal needs to rotate easily. If the gimbal experiences friction and doesn't rotate easily, the cage won't rotate smoothly about the drone. Therefore, the cage won't reduce frictional force as examined in the previous section.

- The diameter needs to be bigger than that of the drone with the propeller on it. Because the ring of the gimbal can rotate, and if the diameter of the ring is not bigger than the drone's 
diameter, the propellers are likely to collide with the ring. As a result, the rotation ability of the ring is disturbed, and the gimbal becomes unable to have a full rotation. Moreover, the propellers can break or the propellers can cut the ring.

- A drone has to carry several components. To let the drone allow to carry as much payload as possible, the weight of the gimbal should be as low as possible.

- It needs to be strong enough so that it does not bend due to the weight of the drone

The drone is $400 \mathrm{~mm}$ long, with the propeller on its longest side. So, the diameter of the outer frame needs to be more than $400 \mathrm{~mm}$. The outer frame was chosen to be $420 \mathrm{~mm}$. Initially, the outer diameter was designed to be circular. However, the most $3 \mathrm{~d}$ printers do not allow printing a model with $400 \mathrm{~mm}$ length. So, the circular outer frame was divided into eight arcs and connected to make the frame. To make the outer frame, Polyethylene terephthalate glycol(PETG) material was used. The reason behind choosing this material is, it is firm, and it has low shrinkage property. An object made with PETG can survive large impacts. However, after printing the arcs and connecting them to make the circular ring, it was observed that the ring bends due to the weight of the drone. So, using the outer frame using this material was not suitable. In order to make the outer cage stronger so that it can sustain the weight of the drone, carbon fiber was chosen as the material for the outer frame. However, it was not possible to make a $3 \mathrm{~d}$ printed circle using carbon fiber due to the unavailability of the required printer. So, instead of a circular outer frame, an octagonal outer ring has been chosen. The edges of the ring are made with a carbon fiber cylinder with an outer diameter of $6 \mathrm{~mm}$. The cylinders are connected using joints made of PETG(Polyethylene Terephthalate Glycol).

As it is clear from the figure 3.4 .2 that, the medium diameter of the octagonal shaped gimbal should be equal or more than the previously chosen circular gimbal's diameter.

The relation between medium diagonal and edge is-

$$
e=a(1+\sqrt{2}))
$$

For our design, $\mathrm{e}=42 \mathrm{omm}$, putting this value is equation $(3.2)$ we obtain, $\mathrm{a}=174 \mathrm{~mm}$. The 


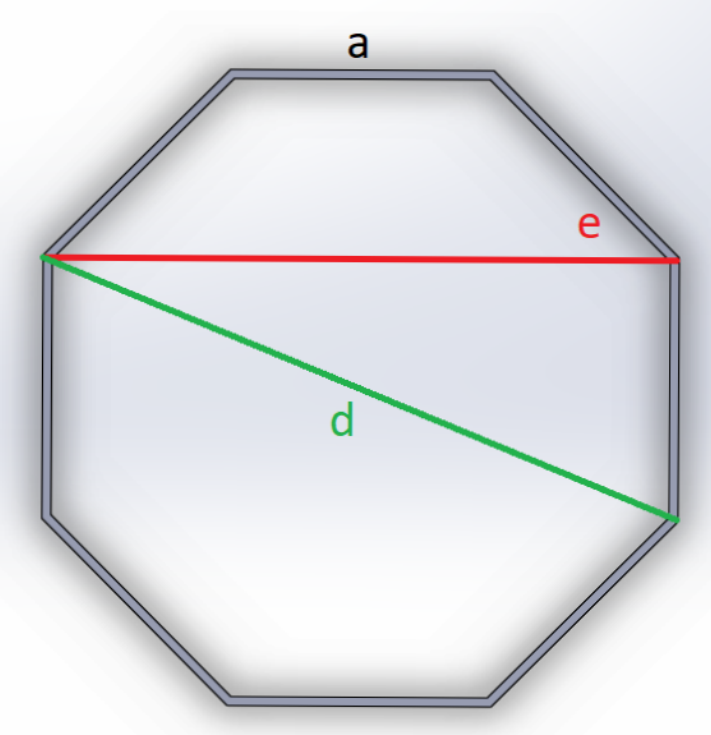

Figure 3.4.2: A regular octagon, where a is edge length, $d$ is long diagonal, and e is medium diagonal.

relation between long diagonal and edge of an octagon is-

$$
d=a \sqrt{4+2 \sqrt{2}}
$$

Putting $a=174$ in equation ( $3 \cdot 3$ ), we obtain, $d=455 \mathrm{~mm}$.

For making a regular octagon, the angle between two edge is kept $135^{\circ}$. In figure $3.4 \cdot 3$, the design of the $135^{\circ}$ joint is shown.

The octagonal gimbal is connected with the cage with two pivoting joints (figure 3.4.1, pivot-4 and 5). A screw connects the cage with this pivoting joint. The screw goes into a bearing that is mounted inside one of the joints in the cage. As a result, the cage has the ability to rotate using this pivoting joint. In figure 3.4.3, the model of pivot is shown. There is a whole in this pivot, so that the edge of the octagonal gimbal can penetrate. 

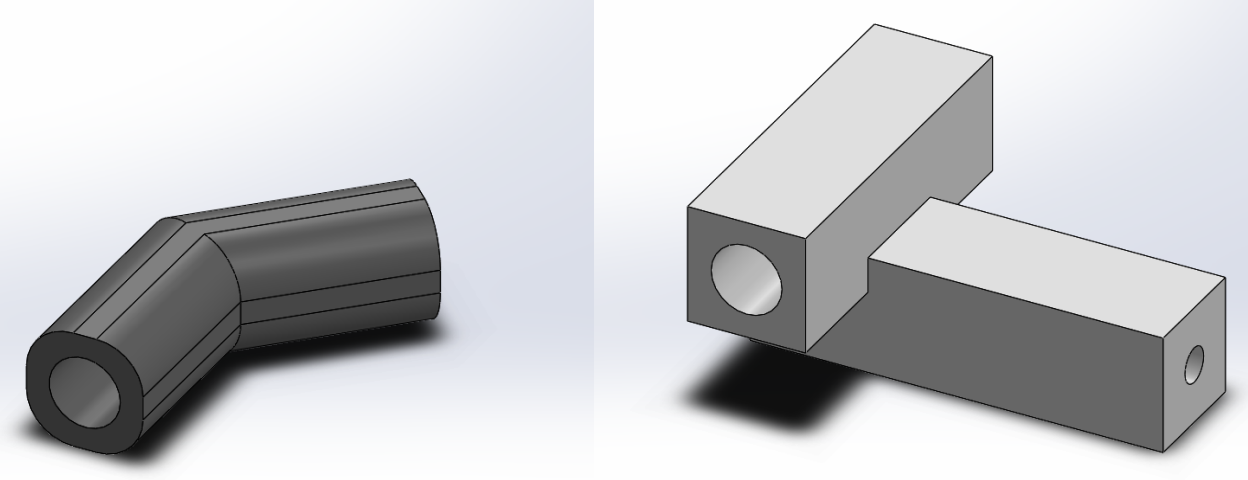

Figure 3.4.3: Left: Joint between the two edges of a octagonal gimbal ring; Right: Pivot that connects the octagonal gimbal with the cage.

The middle gimbal also connect with the inner gimbal using another pair pivot (figure 3.4.1, pivot-2 and 3 ). The shaft of the inner gimbal goes into the bearing inside the pivots. As a result, the shaft can rotate with respect to the middle gimbal (Octagon). The design of this pivot is shown in figure 3.4.4. This pivot also has a hole to let the edge of the octagonal gimbal go through it.

\subsubsection{CAGE}

The most important part of the design is the cage because it protects the entire system that is mounted inside it. So it has to be strong, but there are some constraints in design consideration.

- The size can't be too large. As the size expands, the weight likewise increments. Keeping the weight of the cage as little as possible helps the drone carry more payload and increase flight time.

- The cage has to be perfectly spherical so that it can rotate. If the cage is not spherical, it will not roll smoothly when it comes to in contact with an obstacle. This may cause unwanted bounce, which may disturb the normal flight path. 

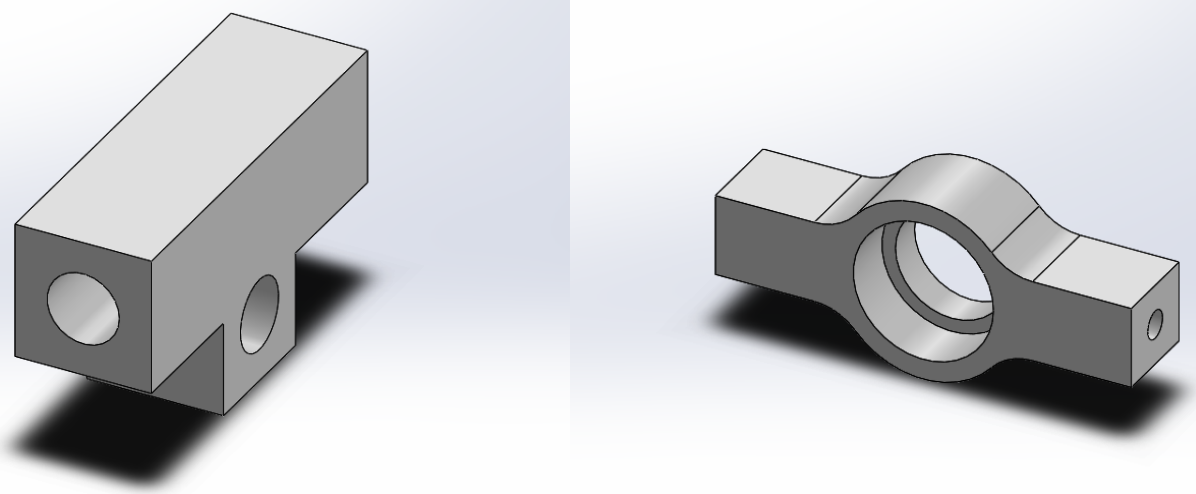

Figure 3.4.4: Left: Pivot that connects the octagonal gimbal with the inner gimbal's shaft; Right: Pivot that connects with the drone.

- In our design, the cage itself works as the outer gimbal. So, it has to be strong enough to survive collision but must not have too much inertia to disturb rotation when a frictional force applies.

- If the collision energy is high, the probability of damage is also high. So, the cage should be able to absorb some collision energy to minimize the probability of damage.

In order to make the cage, the truncated icosahedron shape has been choosen. A truncated icosahedron is a three dimensional polyhedron. It has 32 polygons where 20 polygons are hexagons and the rests are pentagons [33]. The total number of edges is 90 and there are 60 vertices where two hexagons and one pentagon meet in each vertex [33]. This shape is commonly used for soccer balls. The dihedral angles are as follows-

- Hex-Hex angle: 138 degrees, 11 minutes [3]

- Hex-Pent angle: 142 degrees, 37 minutes [3] 
In order to make the cage, 60 joints have been printed to use as the vertices. The joint maintains the dihedral angles' requirement. The joint without the hole for the bearing was found online [1], and then it was modified to place the bearing inside it. Both the joints are shown in figure 3.4.5.

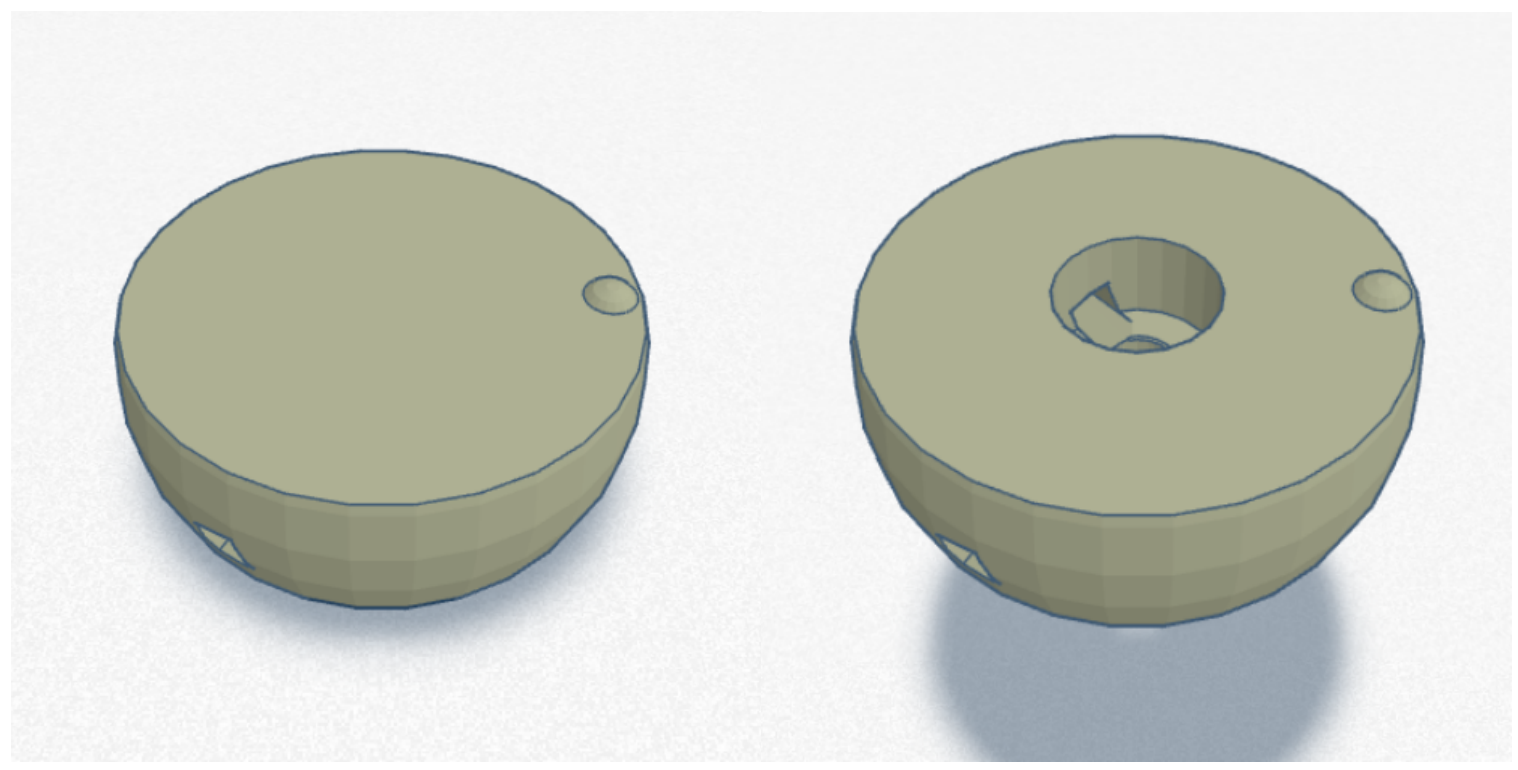

Figure 3.4.5: Joints of the truncated icosahedron shaped cage. Each joint has three holes, where two edges of two different hexagon is inserted, and one edge from pentagon is inserted; The joint in the left has no space holding bearing; The joints in the right has an additional hole where a bearing is mounted.

A truncated icosahedron is an Archimedean solid [33]. The formula [2] for calculating the length of each edge given the radius of the solid is as follows:

$$
r=a / 4 \sqrt{58+18 \sqrt{5}}
$$

where, $r$ is the radius of the shape and $a$ is the edge length. As the desired radius is more than $220 \mathrm{~mm}$ considering the size of the chosen drone, the edge length is $8.9 \mathrm{~mm}$.

The edges were made using carbon fiber tubes. Using carbon fiber makes the cage strong while keeping the weight light. The properties of carbon fiber tubes used in this work in shown in Table 3.4.1. 


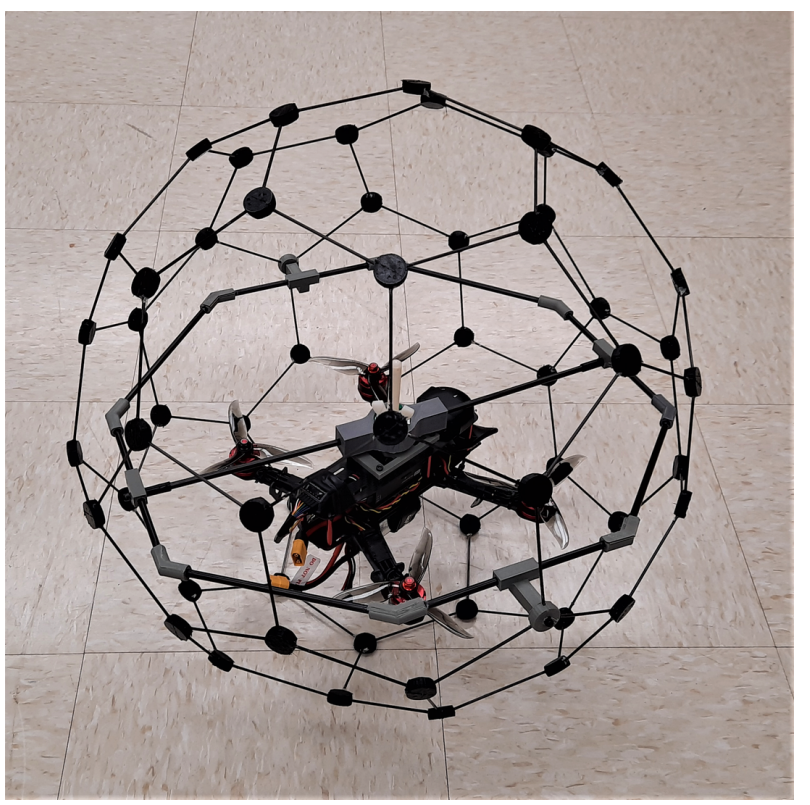

Figure 3.4.6: Fully assembled truncated icosahedron shaped caged drone.

Table 3.4.1: Material properties of carbon fiber tubes used to make the cage.

\begin{tabular}{||cc||}
\hline Property & Description \\
\hline \hline Material & Carbon Fiber \\
\hline Appearance & Plain \\
\hline Shape & Round Tube \\
\hline Texture & Smooth \\
\hline Color & Black \\
\hline Clarity & Opaque \\
\hline Wall Thickness & 0.02” \\
\hline Tolerance Rating & Standard \\
\hline Outer Diameter & $0.078 ”$ \\
\hline Inner Diameter & $0.039 "$ \\
\hline Inner Diameter Tolerance & -0.006 " to $0.006 ”$ \\
\hline
\end{tabular}

Considering the constraint that, the cage should have the ability to absorb some of the collision energy, it is needed that the cage has some sort of flexibility. The edges were made using carbon fiber cylinder which are strong but are not flexible. In order to add some flexibility or elasticity, the 
joints are made using thermoplastic polyurethane (TPU 95A) which is a flexible material.

\subsubsection{Concluding Remarks on the Design}

The cage and gimbal system weighs around $230 \mathrm{~g}$. So, the total weight of the drone, including the cage, is $82 \mathrm{og}$. This meets the desired weight consideration. The diameter of the cage is $480 \mathrm{~mm}$. As the cage joints are made of flexible material, the cage has some elastic property which helps absorb energy during a collision. The edges of the cage are made of carbon fiber cylinders which makes the cage strong. As the outer frame and the inner frame of the gimbal are made of carbon fiber cylinders, the gimbal is also strong and capable of holding the drone's weight without bending. Finally, the bearings in all the pivots help the cage rotate smoothly. So, whenever the drone situated at the center of the cage feels any disturbance due to collision and tends to change the roll/pitch/yaw angle orientation, the cage rotates and keeps the drone in a stable flight condition.

The cage's previous design is good as it can successfully provide protection to the drone and help correct undesired roll, pitch, or yaw angle. However, the design is very complex, and the building process is time-consuming. More importantly, the maintenance of the cage also takes time. For example, the truncated icosahedron shape requires joints that connect two pentagons and one hexagon. If any edge of any polygon breaks after a collision or crash, two joints connected to that

edge need to be changed. Because when the carbon fiber tubes are glued into the joints, the only way to remove them from the joints is to cut the joints. So, the joints have to be disconnected from the edges. To disconnect the joints and the edges is hard as the connections are made as strong as possible for making a safer cage. Also, the cage's diameter has to be larger than the outer gimbal diameter. Otherwise, the outer gimbal cannot rotate freely inside the cage. As a result, the cage's overall size becomes large compared to the drone that the cage protects. As the cage becomes large, the weight increases. Because of these drawbacks, the following design will make the cage smaller than the previous one, which disallows installing a 3-axis gimbal inside the drone.

\subsection{Turtle Shell Shaped Cage}

If the goal is to keep the drone protected from potential collision around it a spherical shaped cage will definately fulfill this goal. However, a spherical cage takes more space than requires to make a fully protected cage. The cage that protects a drone has a diameter larger than the width of the 
drone. If the cage is spherical, there remains a unused space above and below the drone inside the cage as the height of the drone is much smaller than the width. As the spherical cage is inefficient in space consumption, an ellipsoidal cage can be a better option. It is possible to adjust the polar radius and equator radius of the ellipsoid based on the drone's dimension. As the polar radius will depend on the height of the drone, there will be less unused space. For the same radius, an ellipsoidal cage needs smaller space than that of a spherical cage, and it can protect the drone. But, if the cage is made smaller, it is not possible to mount a 3 axis gimbal inside the cage. However, following a crash, there is a higher possibility of the drone falling into the ground upside down. An asymmetric cage can prevent this problem.

\subsection{Design Considerations for Turtle Shell Shaped Cage}

First of all, it is mandatory to protect the drone from all around it to maximize safety. As we are considering to minimize the size, it is not possible to mount a 3 -axes gimbal inside the protective structure. But, it is possible to allow yaw rotation while keeping the size smaller. The cage is given a shape very close to a turtle shell, so that after falling into the ground the cage can roll in the ground to a suitable position to allow the drone can take off again. This section presents the design and fabrication of the turtle shell shaped caged drone. The design consists of the following parts:

- Single axis Gimbal allow the cage rotate freely about the yaw axis. This helps minimize frictional forces acting perpendicular to the drone's yaw axis.

- A cage capable of protecting collision from all directions. If the drone falls into the ground, the cage should help the drone to reorient to a take off position.

\subsubsection{Single AXIS Gimbal}

We have opted out of using a 3-axes gimbal system for this design to minimize the radius of the protective structure. But allowing the protective structure to rotate about the drone's yaw axis will not increase the size of the cage. It is wise to use a protective structure that can rotate freely with respect to the drone because using a rotary is better than a fixed protective structure [30]. 


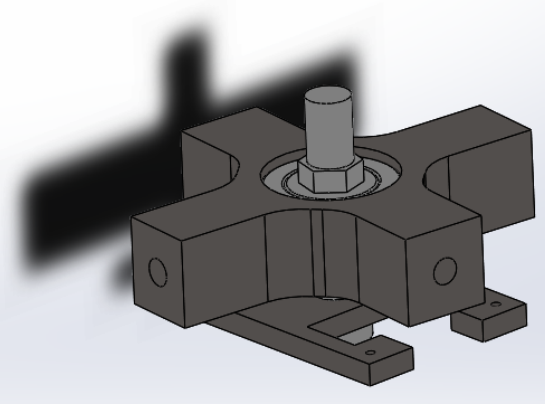

a

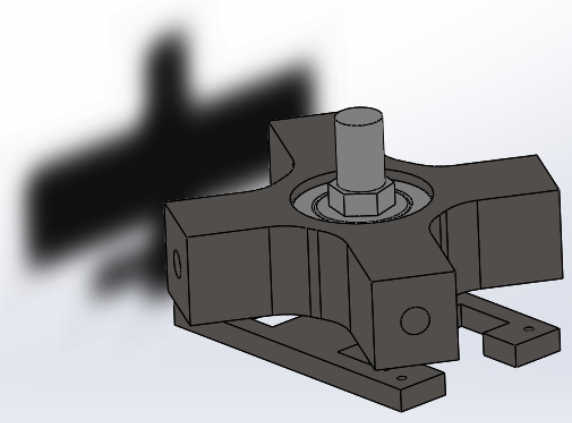

b

Figure 3.6.1: Single axis gimbal; (a), (b) are snapshots of gimbal position with respect to the main frame.

The main part of the gimbal is a central part having four arms. The arms help to connect the cage and the gimbal using carbon fiber shafts. We printed the central part using Ultimaker Cura Printer. The material used for this is PETG. The central part has a hole in the middle where a bearing is mounted. There is a screw, which goes through the mainframe and the bearing hole mounted in the gimbal's central part. A nut has been used to keep the screw tight. As the screw connects both the mainframe and the gimbal, the gimbal can rotate freely without disturbing the mainframe, which holds the drone. Moreover, the gimbal is connected with the cage using carbon fiber shafts. As a result, the cage rotates with the gimbal. Figure 3.6.1 shows random positions when the gimbal rotates. The mainframe stays fixed with the drone.

\subsubsection{CAGE}

The previous cage was of a truncated icosahedron shape (Figure 3.4.6). This shape stays very rigid because every polygon is connected to a number of polygons. These polygons support each other. If any part of a polygon suffers a problem, all the related connections suffer problems as well. If any part of the cage needs to be changed, some related parts also need to be changed. That is why 
the cage should be simpler. This cage consists of ten arms. Each arm consists of five carbon fiber rods connected by $3 \mathrm{~d}$ joints. The two side-by-side arms are not connected. As this cage has fewer interconnected edges, it will not be as rigid as the previous cage.
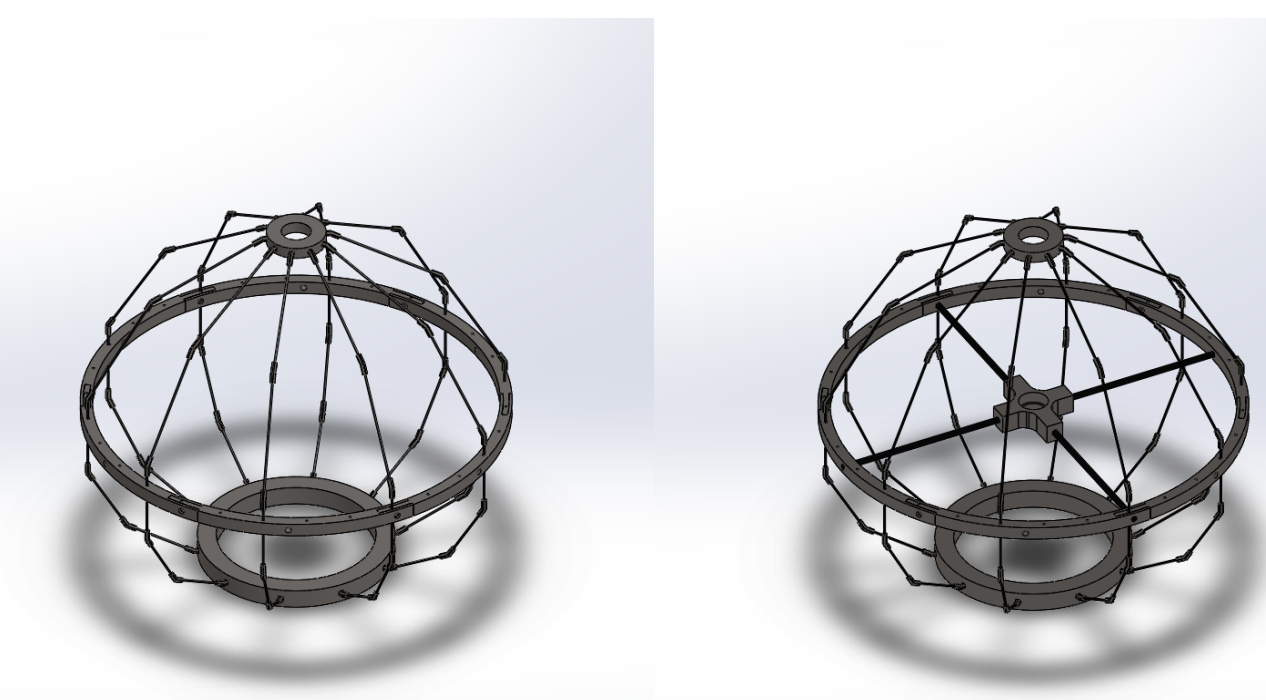

Figure 3.6.2: Left: Turtle shell shaped cage; Right: Turtle shell shaped cage with inner frame is mounted in it.

It is not possible to mount a 3 -axes gimbal system inside the cage, as the radius is smaller than the truncated icosahedron shaped cage. The absence of a gimbal system does not allow the drone to mitigate unwanted roll or pitch. So, it is very probable that after a collision, the drone may lose altitude due to roll/pitch imposed by the impact and may crash into the ground. If the drone falls into the ground, it is required that the drone can take off again. But, if the drone falls in an upsidedown orientation, it will not be able to take off. Our design (Figure 3.6.2) of the cage is inspired by the working principle of turtle-shell, and can roll back to a stable position, like a turtle shell, which allows the drone to take off again.

A turtle shell has a variable radius of curvature. The bottom has smaller radius and the radius increases gradually and become most at a top part of the cage. The cage three major components; (i) joints between the edges, (ii) carbon fiber edges and (iii) circular rings to hold all the edges 


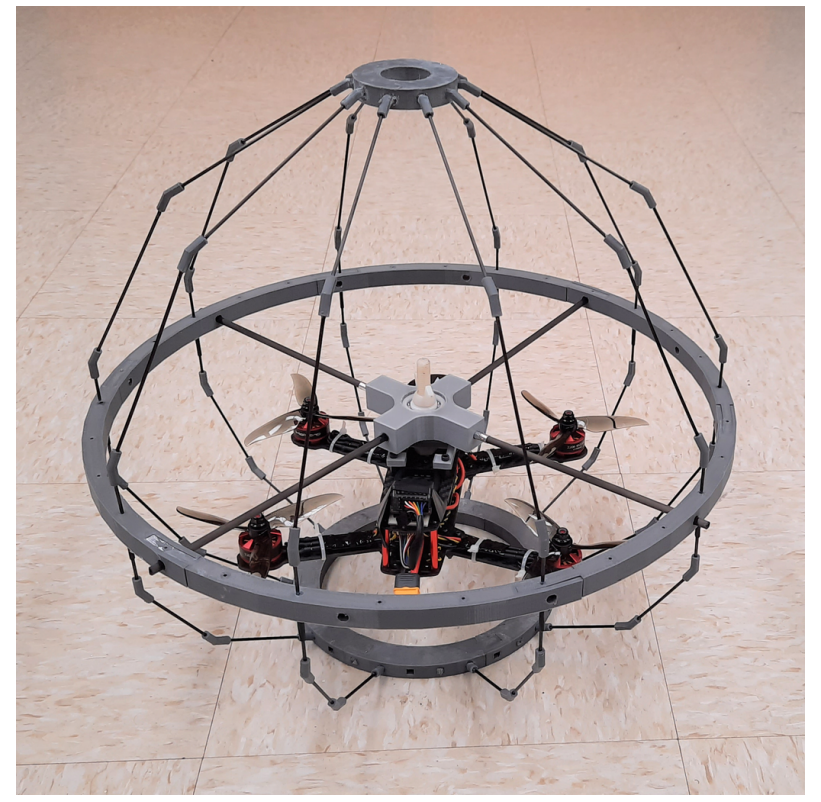

Figure 3.6.3: Fully assembled turtle shell shaped caged drone.

together.

The joints are $3 \mathrm{~d}$ printed. Each joint connects two edges $150^{\circ}$. The carbon fiber rods makes the edges. Two carbon fiber rods connect each other using a $3 \mathrm{~d}$ printed joint. As one edge connects with only one edge using a simple joint, the overall design is simple. The variable radius is made by making the edges of different length. At first ten curves were made using joint and carbon fiber rod. Then these curves are oriented in a equal space in a circle using top, mid and bottom ring of the cage. Figure 3.6.2 shows the cage (left) and the cage with the gimbal mounted in it (right). This system is connected with the mainframe of the drone. The fully assembled turtle shell shaped caged drone is shown in figure 3.6.3.

\subsubsection{Concluding Remarks on the Design}

This cage is smaller than the previous cage. The design is more straightforward. It is effortless to make changes if any component breaks. So, this cage serves the purpose of getting a protective structure with simple maintenance. The two developed caged drones were experimented to analyze their effectiveness. The experiments were not identical because the turtle shell shape caged 
drone does not have gimbal system, and so cannot roll in all directions. In the next chapter, the experimental results will be discussed. 


\section{4 \\ Results}

In the previous chapter, the design criteria, constraints, and design process have been discussed. It is not guaranteed that the design process's assumptions will hold good after the experimental trial. However, based on the experiments, observations, and analyzing data, the design process's drawbacks can be figured out and solved for future improvements. In this chapter, the two cages, designed and developed as described in the previous chapter, were put in several experimental trials to observe their functionality. The two designs have some similarities and some differences. So some of the experiments were identical, and some others were different. In the following sections, we will discuss the experiments' findings for both cages and make related reasonings.

\subsection{Experiment on the Truncated Icosahedron Shaped Caged Drone}

This section describes the experiments performed on the truncated icosahedron shaped caged drone. As this cage's shape is very close to a sphere, and as it has a 3 -axes gimbal mounted inside 
it, it should act as a ball, rolling on the ground, and even along a wall. Moreover, as the cage's primary goal is to survive collision, it should keep the drone safe after a strong impact. The following experiments were performed to observe the effectiveness of the cage.

For further references go to the next url: https://www.youtube.com/watch?v=wXr7xnFbTHg\& list=PLR_ovgr_c8EQvAiRfXq2-CCqNWmKDVvrn\&index $=1$

\subsubsection{RolLING IN THE GROUND}

Due to the cage's sphericity coupled with the 3-axes gimbal system, flying the drone close to the ground should cause the cage to roll on the ground. To confirm this hypothesis, we performed a couple of experimental flights. Figure 4.1.1 shows some snapshots from the experiment. The drone starts from the position shown in figure 4.1.1 (a) and move to another position shown in figure 4.1.1 (b). It was confirmed that the cage works as a wheel when the drone is in the ground. When the cage rotated on a flat surface, the robot could move smoothly; but the rough surface caused the drone to bounce. The cage could survive jumping and rolling on several surfaces.
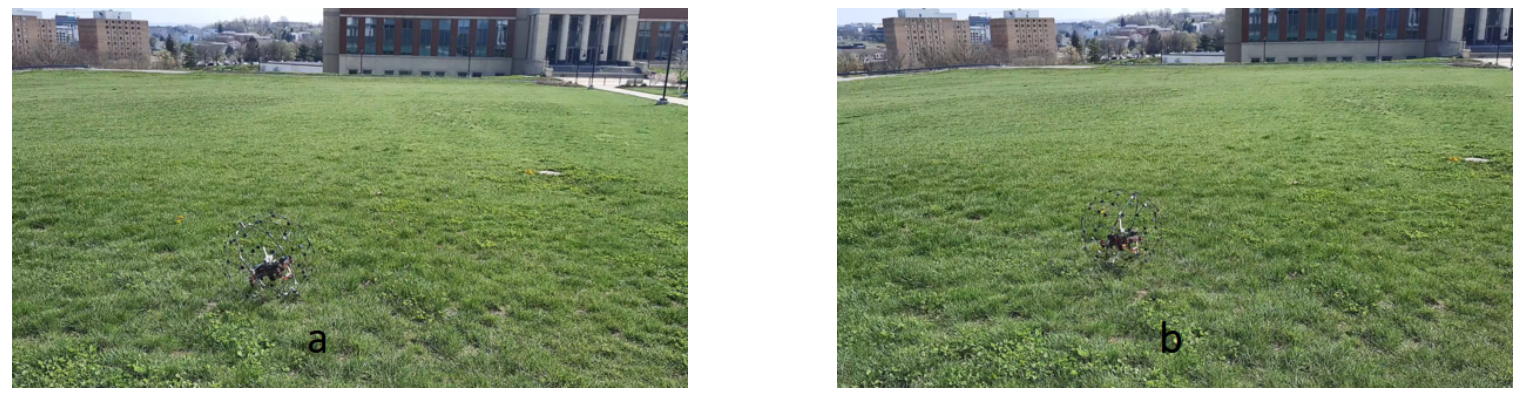

Figure 4.1.1: Snapshots when the drone is rolling on the ground.

\subsubsection{Climbing Up and Down a Wall}

After confirming the drone could successfully roll on the ground, it was expected that the drone could roll along a wall. To test this, the drone's flight trajectory was planned close to the wall so that the cage could contact with it. It was observed that the wall's smooth surface enabled an effortless roll compared to the ground roll. Figure 4.1.2 shows some snapshots when the drone climbed up and down a wall. The drone starts from the position shown in figure 4.1.2(a) and move to another 

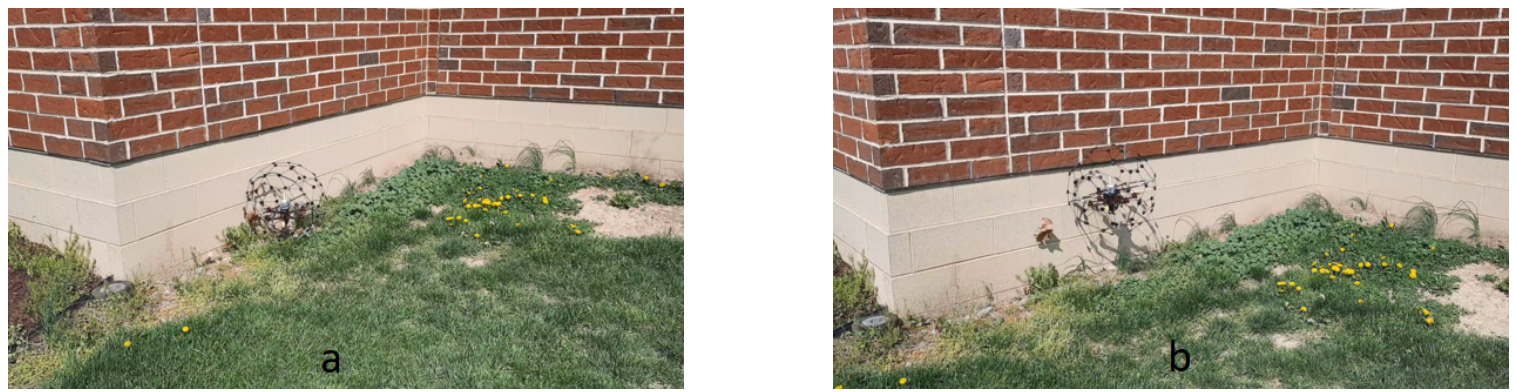

Figure 4.1.2: Snapshot of the truncated icosahedron shaped caged drone when it is rolling along a wall.

position shown in figure 4.1.2 (b). While climbing up and down the wall, the drone continuously bounced with the wall because it was hard to maintain a specific distance from the wall by controlling the drone manually. The cage survived those minor collisions with the wall, so it was easier to decide the next experiment of performing a harder collision.
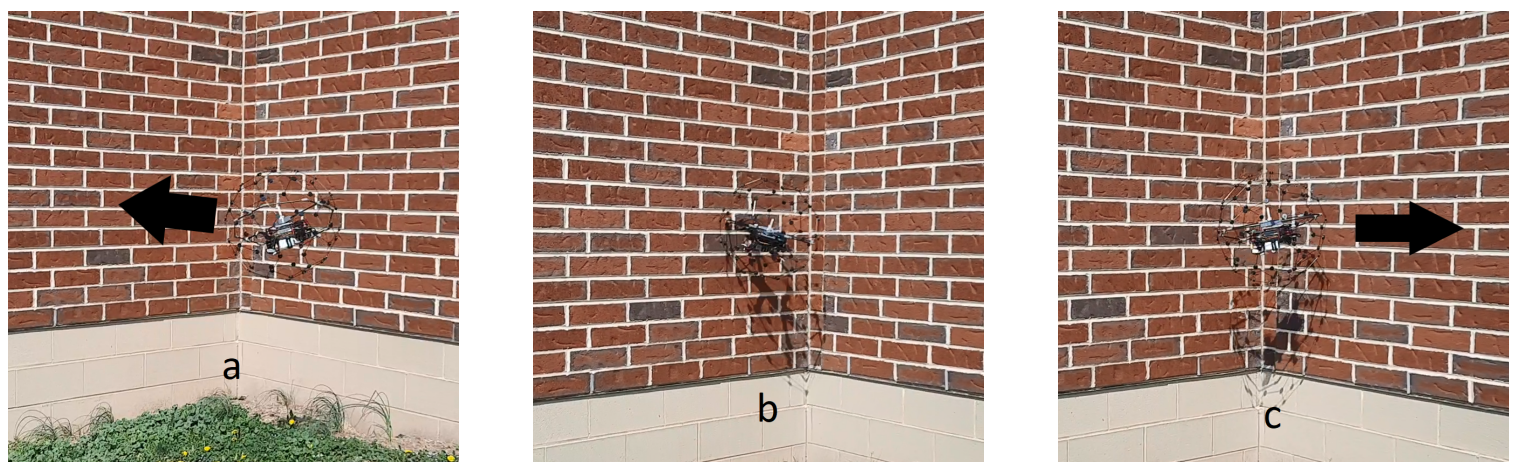

Figure 4.1.3: The truncated icosahedron shaped caged drone collides with a wall. In figure (a) the drone is moving towards the wall, in figure (b) the collision is happening, and in figure (c) the drone is bouncing back from the wall.

\subsubsection{COLLIDING With THE WALL}

The most crucial part of the experiment was to confirm if the cage could survive a significant collision with objects in the environment. As shown in figure 4.1.3, the drone was flown into the wall at an average speed of $6 \mathrm{~km} / \mathrm{h}$, and it was observed that the drone's cage absorbed energy from the 
collision and bounced away from the wall.

\subsection{Flight Data Analysis on the Truncated Icosahedron Shaped Caged Drone}

It is essential to understand how the critical parameters respond after attaching the drone to a cage that can rotate. The flight data was analyzed to get a better understanding, and the responses of the parameters were observed. Figure 4.2.1 shows the roll angles with respect to time.

In the plot, the region marked in the blue rectangle represents the state when the drone is rolling on the ground. The drone was moved in a particular direction by changing the roll angle while keeping the altitude low so that the cage stays in contact with the ground. In this case, a frictional force acts, as shown in figure 1.2.1. So, the drone should have experienced unwanted rotation. But, we can observe from the figure that the roll setpoint and estimated roll are the same. The roll setpoint here is the command given by the pilot, and roll estimated is the actual roll experienced by the drone. The estimated roll follows the roll setpoint smoothly because the cage rotates due to the frictional forces, and the drone is not affected by the frictional forces. The region marked in a black rectangle in the plot represents the state when the drone is flying. In this state, the user's command is minimal, and the roll estimated follows the roll set by the user.

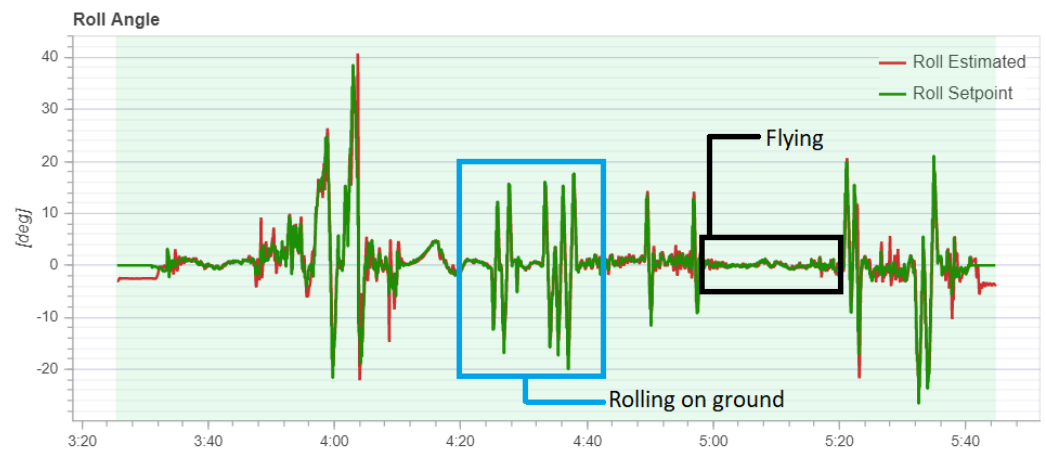

Figure 4.2.1: Changes in roll angle with time. The green curves shows the roll set by the pilot; the red curves shows the roll estimated by the drone. $\mathrm{Y}$ axis of the plot shows roll angles in degrees. $\mathrm{X}$ axis shows time in minutes. 


\subsection{Experiment on the Turtle Shell Shaped Caged Drone}

This section describes the experiments performed on the turtle shell shaped caged drone. This cage's shape is not spherical. It does not have a 3-axes gimbal mounted in it as well. So, this cage cannot roll like the first cage. However, the cage can rotate about the yaw axis, enabling the drone to roll along a wall horizontally. Moreover, the cage's principal objective is to protect the drone after a collision, so it should survive collision forces. As the cage has limitations mitigating unwanted roll or pitch angles due to the lack of a 3 -axes gimbal system, the drone can crash following a collision. But, since the shape is similar to a turtle shell, and so, the cage should have the ability to reorient itself in a suitable take-off position. So, the following experiments were performed to observe the effectiveness of the cage.

For further references go to the next url: https://www.youtube.com/watch?v=vklc9kWLjdU\& list=PLR_ovgr_c8ESBbbF4RPXfzdOGg8Awh7-g\&index $=1$

\subsubsection{Hitting The WaLl}

We tested if the cage could survive collision with the wall. It was observed that after the impact, the drone lost some altitude, as shown in figure 4.3.1. This happened because, after the collision, the roll and pitch angle changed to a significant amount.
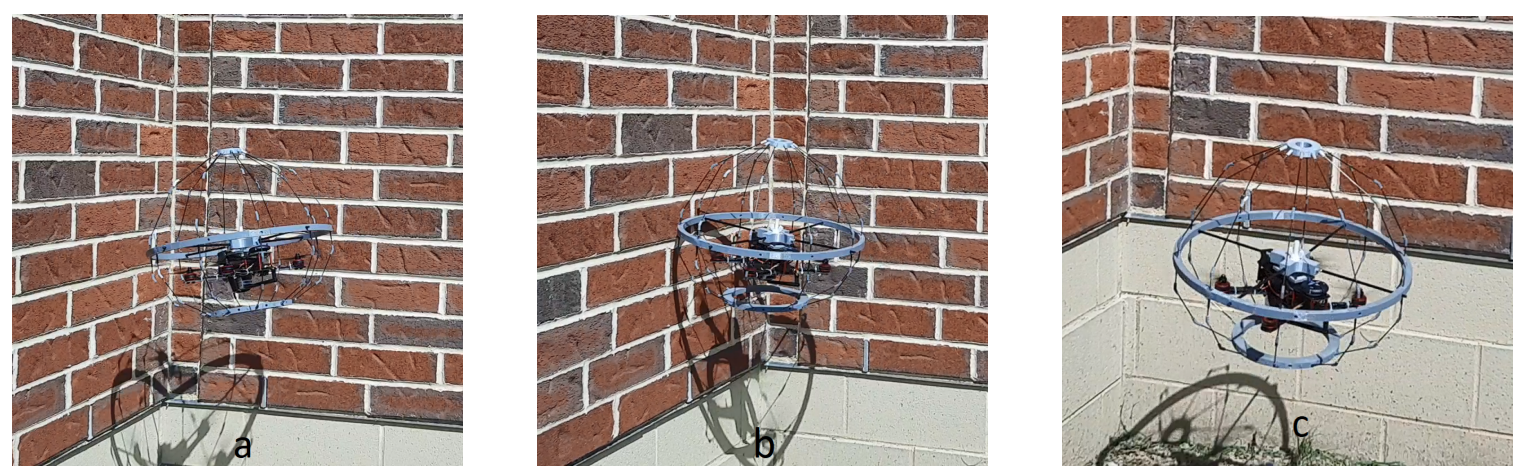

Figure 4.3.1: The truncated icosahedron shaped caged drone collides with a wall. In figure (a) the drone is moving towards the wall, in figure (b) the collision is happening, and in figure (c) the drone is bouncing back from the wall. 


\subsubsection{Reorient to Take-Off Position}

It was chosen in the design criteria that the cage will not have rotation ability-the inability to rotate results in the drone's unwanted roll or pitch angle after a collision, which causes its loss of altitude. As a result, the drone will probably crash into the ground following a collision. It is then needed to make sure that the drone does not fall in an upside-down state. So, the design of the cage was inspired by the turtle shell. A turtle can reorient itself with the help of its shell [11]. The idea was to give the cage a shape similar to a turtle shell so that whenever the cage falls into the ground, its shape helps the drone to reorient itself in a suitable take-off position.
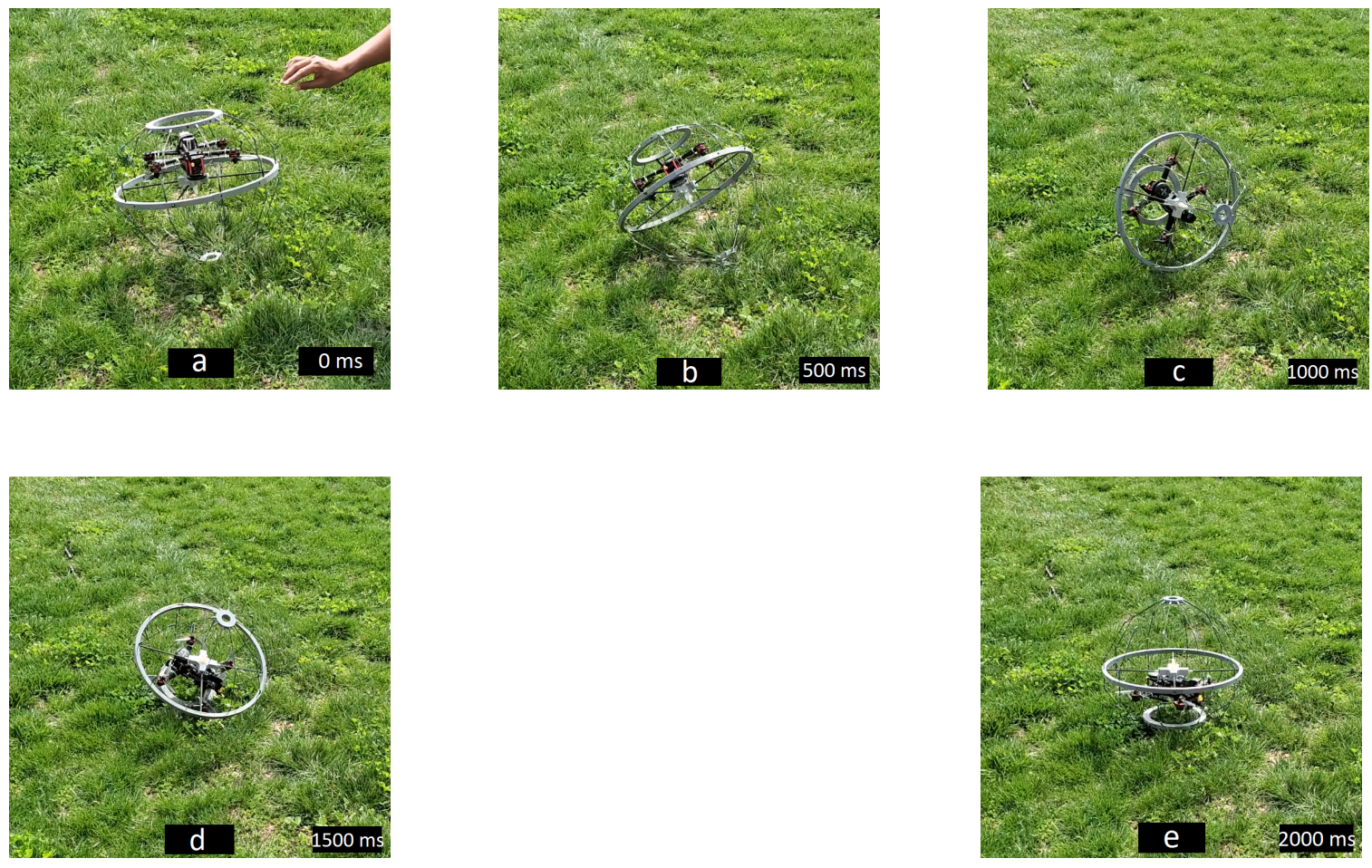

Figure 4.3.2: Drone with turtle shaped cage reorient to take-off position. The reorientation progress through time in alphabetical order shown in the figure. The time step is also mentioned in the figure.

So it was important to observe whether the drone can reorient itself to a take-off position. We put the cage upside-down and leave the cage. The cage could roll and successfully orient to a stable 
state from where the drone could fly. In figure 4.3.2, some snapshots of this experiment have been shown. A similar experiment was performed indoors on a flat surface. The cage could reorient as it did outside on the grass.

\subsection{Flight Data Analysis on the Turtle Shell Shaped Caged Drone}

The experiments were executed successfully. So, it was needed to understand the behavior by observing the flight data. Figure 4.4.1 shows the changes in roll angle with respect to time. The area marked in a black rectangular box represents the state when the drone is flying. We can observe from the plot that, during flying, the roll setpoint is very high. The cage rotates about the yaw axis only; when the drone needs to be moved by changing roll, it is hard for the drone to roll due to the inertia of the cage. So, the roll setpoint needs to be high for a slight change in roll in practice. This could be why a protective structure is kept very light when it can only rotate about one axis.

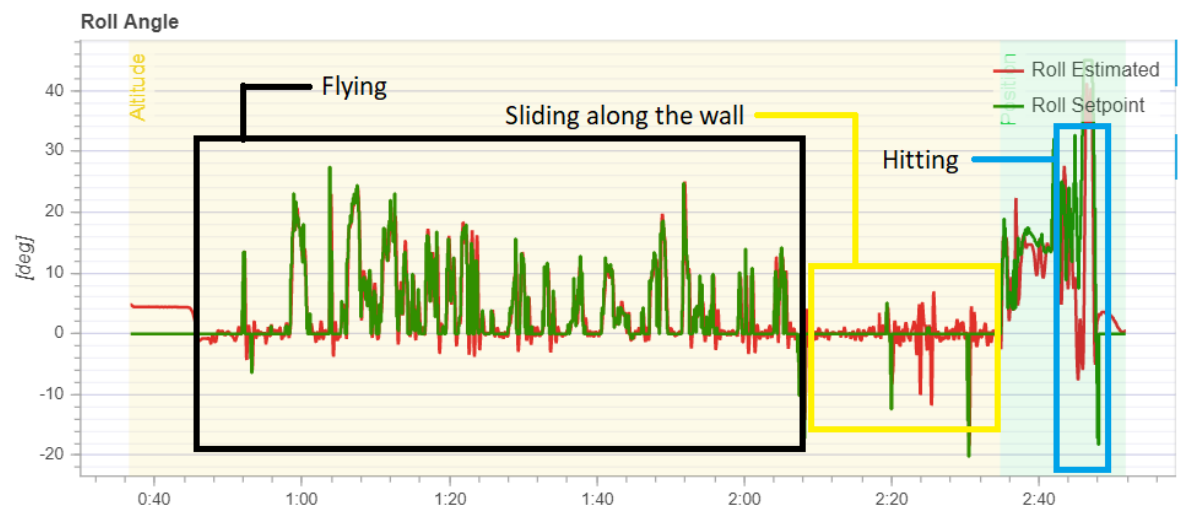

Figure 4.4.1: Flight data of turtle shell shaped caged drone obtained from pixhawk mini 4 . The $\mathrm{X}$ axis represents time in minutes, and the $\mathrm{Y}$ axis represents angle in degrees. The green curves are roll angles set by the user, the red curves are roll estimated by the drone.

The area marked using the yellow rectangular area in figure 4.4.1 represents when the drone was sliding with the wall. The roll setpoint was kept zero most of the time, but we can observe some significant estimated roll in the plot because when the drone was moving along the wall, the minor collisions caused unwanted roll. When the drone hits the wall, the state of the roll angle is shown in the blue rectangular mark. It is observed that when the collision occurs, the estimated roll has 
a negative value where the roll set by the pilot was positive. The estimated roll became negative because the frictional forces after collision rotate the drone in the opposite direction.

\subsection{COMPARISON}

A comparison between the two developed drone is shown below:

Table 4.5.1: Comparison between the truncated icosahedron shaped caged drone and turtle shell shaped caged drone.

\begin{tabular}{|l|l|l|}
\hline Characteristics & $\begin{array}{l}\text { Truncated Icosahe- } \\
\text { dron Shaped caged } \\
\text { drone }\end{array}$ & $\begin{array}{l}\text { Turtle Shell Shaped } \\
\text { caged drone. }\end{array}$ \\
\hline Weight & $820 \mathrm{~g}$ & $850 \mathrm{~g}$ \\
Diameter & $480 \mathrm{~mm}$ & $440 \mathrm{~mm}$ \\
Height & $\mathrm{N} / \mathrm{A}$ & $380 \mathrm{~mm}$ \\
Cost & $\$ 110$ & $\$ 52$ \\
Assembling time & 32 hours & 8 hours \\
Propeller Protection & yes & yes \\
Maintenance & hard & yedium \\
Mitigation of un- & Roll, pitch, yaw & \\
wanted rotation & & yes \\
Ability to take off after & yes & \\
crash & & \\
\hline
\end{tabular}

\subsection{Concluding Remarks}

The rotary protective structure minimizes collision forces when an oblique collision happens. The truncated icosahedron shaped cage can rotate about all three axes, so this protective structure minimizes collision forces for all possible oblique collisions. The turtle shell shaped cage can only rotate about the yaw axis. This protective structure can reduce collision forces when an oblique collision occurs in the XY plane. So, a truncated icosahedron shaped cage is more efficient in minimizing collision forces. As the turtle shell shaped cage does not rotate about roll and pitch axes, it was challenging to perform rolling and pitching due to the inertia of the cage. From this observation, we can 
understand why only a circular protective structure is used when rotation about only the yaw axis is chosen. The manufacturing process of the first one is very complicated and time-consuming compared to the other. The cost of the truncated icosahedron shaped cage is higher than the turtle shell shaped cage. But, it is possible to reduce manufacturing costs for both cages. The size of the turtle shell shaped cage is less than the first one. Despite having several differences, both the cage can survive collisions and can protect the drone. Both the cages can reorient to a take-off position after falling into the ground. It is always better to have rotation ability to mitigate unwanted rotations due to a collision. But whether there is any need for rotation ability will depend on the mission objective. If the requirement is low cost and less complex design, then the turtle shell shaped cage would be suitable. Otherwise, a truncated icosahedron shaped caged drone is a better solution for caged drones. 


\section{5 Conclusions And Future Work}

\subsection{CONCLUSIONS}

This work has dealt with the design and manufacturing of two protective structures for quadrotor drones. Particular attention has been paid to understand the consequences of a quadrotor drone's collision with a solid obstacle. As there are several consequences, it was hard to reach an ideal solution. However, it was easy to understand that collision outcomes are dangerous for a quadrotor drone. As a collision can break parts of the quadrotor drone, the first and foremost requirement was to guarantee a protective mechanism for the drone's components. While trying to develop a strong protective structure, it was also essential to keep the protective structure's weight as low as possible so that a drone can carry the cage easily. Another outcome of a collision is introducing unwanted rotation to the drone, which often leads to loss of thrust. The drone experiencing this collision is likely to crash into the ground. So, it was important for the protective structure to mitigate unwanted rotation to avoid a crash. It was also important to consider that following a collision, 
if a drone crashes, the drone should take off again.

Based on the two considerations discussed above, the first protective structure was designed and built. This protective structure has a truncated icosahedron shape. This shape is almost spherical and protects the drone from all directions. As the shape is very close to a sphere, the cage could be given the ability to rotate around the drone. So, a 3-axes gimbal has been mounted in between the cage and the drone. With the help of the gimbal, the cage can rotate around the drone. This rotation ability reduces unwanted rotation of the drone introduced by a collision, while the truncated icosahedron shaped cage protects the drone from breaking after a collision. Also, if the drone crashes, the 3-axes gimbal helps the drone stay in the normal position to take off again. Most of the parts of the cage were made of carbon fiber rods. The rods are joined using $3 \mathrm{D}$ printed joints. TPU95 material was used to make these joints. To make the 3 -axes gimbal, both ${ }_{3} \mathrm{D}$ printed parts and carbon fiber rods were used. The inner gimbal is mounted at the top of the drone, and it rotates about the drone's yaw axis. This inner gimbal has a carbon-fiber shaft that connects the middle gimbal with the inner gimbal. The middle gimbal is an octagonal ring mounted with the inner gimbal's shaft and can rotate about the shaft axis. The outer gimbal is the cage, which is mounted with the middle gimbal. This outer gimbal can rotate about the middle gimbal. The rotation axis is perpendicular to both the drone's yaw axis and the rotation axis of the middle gimbal. This design meets the requirement ideally, but it has some drawbacks as well. The manufacturing process is challenging for this shape. Moreover, after a collision, if any part of the cage breaks, it is hard to rebuild that particular part, as each piece is connected with some other parts.

The use of a 3-axes gimbal was inspired by the work shown in [7]. In [7], three circular rings have been mounted orthogonal to each other to assemble a 3 -axes gimbal. The drone is mounted on a platform that is connected with the inner gimbal using a shaft. The shaft is fixed with the inner gimbal, and when the inner gimbal rotates, the shaft rotates with it. In our design, we did not use three circular rings to form the 3 -axes gimbal. Instead, we used some parts of the protective structure as part of the gimbal system. Instead of making the cage fixed with the outer gimbal, we replaced the outer gimbal with the cage. So, the cage itself works as the outer gimbal and can rotate about the middle gimbal. The middle gimbal is an octagonal ring. It rotates about the inner gimbal. The inner gimbal in the developed design is not a circular ring like that of [ 7$]$. The shaft connected with the inner frame itself works as the inner gimbal and rotates about the drone. A comparison 
Table 5.1.1: Comparison between Gimball [7] and our developed work.

\begin{tabular}{|c|c|c|}
\hline Characteristics & Gimball & $\begin{array}{l}\text { Truncated Icosahe- } \\
\text { dron shaped caged } \\
\text { drone }\end{array}$ \\
\hline Configuration & Coaxial & Quadrotor \\
\hline Control surfaces & Present & Do not required \\
\hline Gimbal system & 3 circular rings & 1 octagonal ring \\
\hline Cage & $\begin{array}{l}\text { Fixed with the outer } \\
\text { gimbal }\end{array}$ & It is the outer gimbal \\
\hline Inner shaft & $\begin{array}{l}\text { Fixed with the inner } \\
\text { gimbal }\end{array}$ & It is the inner gimbal \\
\hline Propeller Protection & yes & yes \\
\hline Materials & $\begin{array}{l}\text { Carbon fiber tube, } \\
\text { ABS }\end{array}$ & $\begin{array}{l}\text { Carbon fiber tube, } \\
\text { TPU95 }\end{array}$ \\
\hline $\begin{array}{l}\text { Mitigation of un- } \\
\text { wanted rotation }\end{array}$ & Roll, pitch, yaw & Roll, pitch, yaw \\
\hline $\begin{array}{l}\text { Ability to take off after } \\
\text { crash }\end{array}$ & yes & yes \\
\hline
\end{tabular}

between the work presented in [7] and our developed work is given in table 5.1.1.

The second protective structure's goal was to protect the drone while keeping the design and manufacturing process more straightforward. To make the building process easier, we opted out of using a 3-axes gimbal for this design. As a result, the protective structure would not be able to mitigate unwanted rotation acted on the drone due to collision and thus could fall. It is essential to mention that this protective structure can rotate about the drone's yaw axis, but this rotation does not mitigate unwanted roll or pitch rotation. As it is probable that the cage could crash following a collision, it was important that after a crash, the drone could take off again. To enable this ability, this protective structure was designed inspired by a turtle shell. Like a turtle shell [11], this protective structure can roll to a natural state from any other state. So, after a collision, if the cage falls into the ground upside-down, the cage can roll, place the drone's motors directed towards the top, and the drone can take off again. The model consists of ${ }_{3} \mathrm{D}$ printed parts and carbon fiber rods.

The protective structure could be made fixed with the drone. But we chose to make the cage rotary because $[30]$ proved that a rotary protective structure is better in reducing oblique collision 
forces. Although the idea of making a rotary protective structure was inspired by "Miura-oRing" [30], our developed design has some differences. First of all, the "Miura-oRing" has an origamiinspired protective structure that helps reducing collision forces. Our design does not have any elasticity to minimize normal collision force. So, when a normal collision occurs, the "MiuraoRing" has a clear advantage over our design. But, when an oblique collision occurs, both "MiuraoRing" and our developed cage can rotate and minimize collision forces. The protective structure in "Miura-oRing" only protects when the drone is moving on the lateral axis. But our developed protective design can protect the drone from collision from all directions. If the drone falls to the ground after a collision, the "Miura-oRing" structure does not control the drone's orientation. So, if the drone falls upside-down, the drone stays upside-down until any person reorients the drone to its normal position. But, our developed cage has a turtle shell like shape, which helps the drone reorient itself if it falls upside down. So, the drone does not need human assistance to take off again after a crash. A comparison between the work presented in [30] and our developed work is given in table 5.1.2.

Table 5.1.2: Comparison between Miura-oRing [30] and our developed work.

\begin{tabular}{|c|c|c|}
\hline Characteristics & Miura-oRing & $\begin{array}{l}\text { Turtle Shell shaped } \\
\text { caged drone }\end{array}$ \\
\hline Configuration & Quadrotor & Quadrotor \\
\hline Protective structure & Ring & Cage \\
\hline $\begin{array}{l}\text { Rotary } \\
\text { structure }\end{array}$ & About yaw axis & About yaw axis \\
\hline Propeller Protection & Horizontal collisions & All directions \\
\hline $\begin{array}{l}\text { Cushion over the pro- } \\
\text { tective structure }\end{array}$ & Yes & No \\
\hline $\begin{array}{l}\text { Ability to take off after } \\
\text { crash }\end{array}$ & No & yes \\
\hline
\end{tabular}

\subsection{FUTURE WORK}

The models developed in this work are prototypes. There could be many improvements possible. Some steps can be followed for future works that are as follows: 
- The $3 \mathrm{~d}$ printed joints for the truncated icosahedron shaped cage are too big, which adds extra weight. The joints could be made smaller to reduce overall weight but at the same time, should be able to resist to some strong impacts.

- The octagonal middle gimbal rotates about the shaft axis that is mounted in the inner gimbal. This rotation is possible as the middle gimbal is connected with the shaft using a pivot. This pivot has to carry the weight of the drone, including the inner gimbal. So, this is prone to break, and so, needs to be stronger.

- The turtle shell shaped cage is smaller but weighs more because it has more $3 \mathrm{~d}$ printed parts than the truncated icosahedron shaped cage. The middle and the bottom rings can be replaced using carbon fiber octagonal rings. This can minimize weight and increase strength.

- Instead of using joints and carbon fiber rods, aluminium sheet or balsa sheets can be cut into a suitable shape to make the parts of the turtle shell shaped cage. Because the joints where the carbon fiber rods connect each other are $3 \mathrm{~d}$ printed, and fragile.

- The turtle shell shaped caged drone is designed to rotate and place the drone in a suitable position to enable taking-off. If the cage does not roll, a small push toward the drone's yaw axis helps the cage to rotate. This is how a turtle rotates when stuck upside-down. However, the pixhawk controller does not allow the drone to start when the drone is not placed parallel to the horizontal plane. The pixhawk needs to be reprogrammed so that the drone can start from any orientation.

- The drones are difficult to control indoor because the cages obstruct the optical flow sensor's view, the sensor cannot work properly. But, this problem can be solved by programming the optical flow functionality so that the sensor can ignore the rotating cage while calculating distance and velocity.

- The drones can be made autonomous. 


\section{References}

[1] Truncated Icosahedron Bucky Node joints. http://web.archive.org/web/ 20080207010024/https://www.thingiverse.com/thing:629977. Accessed: 202 1-04-15.

[2] Truncated Icosahedron Calculator. http://web.archive.org/web/20080207010024/ https://rechneronline.de/pi/truncated-icosahedron.php. Accessed: 2021o4-15.

[3] Truncated Icosahedron, properties of the truncated icosahedron: Number of faces, edges and dihedral angle measure. http://web.archive. org/web/20080207010024/https://www . coolmath.com/reference/ polyhedra-truncated-icosahedron. Accessed: 2021-04-15.

[4] Parastoo Abtahi, David Y Zhao, Jane L E, and James A Landay. Drone near me: Exploring touch-based human-drone interaction. Proceedings of the ACM on Interactive, Mobile, Wearable and Ubiquitous Technologies, 1(3):1-8, 2017.

[5] Reem Ashour, Tarek Taha, Fahad Mohamed, Eman Hableel, Yasmeen Abu Kheil, Malak Elsalamouny, Maha Kadadha, Kasturi Rangan, Jorge Dias, Lakmal Seneviratne, et al. Site inspection drone: A solution for inspecting and regulating construction sites. In 2016 IEEE 59th International Midwest Symposium on Circuits and Systems (MWSCAS), pages 1-4. IEEE, 2016.

[6] Russell SA Brinkworth and David C O'Carroll. Robust models for optic flow coding in natural scenes inspired by insect biology. PLoS Comput Biol, 5(11):e1000555, 2009.

[7] Adrien Briod, Przemyslaw Kornatowski, Jean-Christophe Zufferey, and Dario Floreano. A collision-resilient flying robot. Journal of Field Robotics, 3 1(4):496-509, 2014.

[8] Pierre-Jean Bristeau, François Callou, David Vissiere, and Nicolas Petit. The navigation and control technology inside the ar. drone micro uav. IFAC Proceedings Volumes, 44(1):1477$1484,2011$. 
[9] Fernando Cunha and Kamal Youcef-Toumi. Ultra-wideband radar for robust inspection drone in underground coal mines. In 2018 IEEE International Conference on Robotics and Automation (ICRA), pages 86-92. IEEE, 2018.

[10] Gareth Dicker, Fiona Chui, and Inna Sharf. Quadrotor collision characterization and recovery control. In 2017 IEEE International Conference on Robotics and Automation (ICRA), pages 5830-5836. IEEE, 2017.

[11] Gábor Domokos and Péter L Várkonyi. Geometry and self-righting of turtles. Proceedings of the Royal Society B: Biological Sciences, 275(1630):11-17, 2008.

[12] Christopher J Drol, Emily B Kennedy, Bor-Kai Hsiung, Nathan B Swift, and Kwek-Tze Tan. Bioinspirational understanding of flexural performance in hedgehog spines. Acta biomaterialia, 94:553-564, 2019.

[13] Davide Falanga, Kevin Kleber, Stefano Mintchev, Dario Floreano, and Davide Scaramuzza. The foldable drone: A morphing quadrotor that can squeeze and fly. IEEE Robotics and Automation Letters, 4(2):209-216, 2018.

[14] Slawomir Grzonka, Giorgio Grisetti, and Wolfram Burgard. A fully autonomous indoor quadrotor. IEEE Transactions on Robotics, 28(1):90-100, 2011.

[15] Shafkat Islam and Abolfazl Razi. A path planning algorithm for collective monitoring using autonomous drones. In 2019 53rd Annual Conference on Information Sciences and Systems (CISS), pages 1-6. IEEE, 2019.

[16] Adam Klaptocz, Adrien Briod, Ludovic Daler, Jean-Christophe Zufferey, and Dario Floreano. Euler spring collision protection for flying robots. In 2013 IEEE/RSJ International Conference on Intelligent Robots and Systems, pages 1886-1892. IEEE, 2013.

[17] Przemyslaw M Kornatowski, Stefano Mintchev, and Dario Floreano. An origami-inspired cargo drone. In 2017 IEEE/RSJ International Conference on Intelligent Robots and Systems (IROS), pages 6855-6862. IEEE, 2017.

[18] Przemyslaw Mariusz Kornatowski, Mir Feroskhan, William J Stewart, and Dario Floreano. A morphing cargo drone for safe flight in proximity of humans. IEEE Robotics and Automation Letters, 5(3):4233-4240, 2020.

[19] GL Leandri, A Sunderland, J Winterflood, and L Ju. Six degrees of freedom vibration isolation with euler springs. Review of Scientific Instruments, 92(2):025 122, 2021. 
[20] Tong Li, Youmin Zhang, and Brandon W Gordon. Passive and active nonlinear fault-tolerant control of a quadrotor unmanned aerial vehicle based on the sliding mode control technique. Proceedings of the Institution of Mechanical Engineers, Part I: Journal of Systems and Control Engineering, 227(1):12-23, 2013.

[21] Sven Mayer, Lars Lischke, and Paweł W Woźniak. Drones for search and rescue. In 1st International Workshop on Human-Drone Interaction, 2019.

[22] Kimberly McGuire, Guido De Croon, Christophe De Wagter, Karl Tuyls, and Hilbert Kappen. Efficient optical flow and stereo vision for velocity estimation and obstacle avoidance on an autonomous pocket drone. IEEE Robotics and Automation Letters, 2(2):1070-1076, 2017.

[23] Stefano Mintchev, Sébastien de Rivaz, and Dario Floreano. Insect-inspired mechanical resilience for multicopters. IEEE Robotics and automation letters, 2(3):1248-1255, 2017.

[24] Yash Mulgaonkar, Gareth Cross, and Vijay Kumar. Design of small, safe and robust quadrotor swarms. In 2015 IEEE international conference on robotics and automation (ICRA), pages 2208 2215 . IEEE, 2015.

[25] Anurag Ranjan, Joel Janai, Andreas Geiger, and Michael J Black. Attacking optical flow. In Proceedings of the IEEE/CVF International Conference on Computer Vision, pages 2404-2413, 2019.

[26] James F Roberts, Timothy Stirling, Jean-Christophe Zufferey, and Dario Floreano. Quadrotor using minimal sensing for autonomous indoor flight. In European Micro Air Vehicle Conference and Flight Competition (EMAV2007), number CONF, 2007.

[27] Chelsea M Sabo, Alex Cope, Kevin Gurney, Eleni Vasilaki, and James Marshall. Bio-inspired visual navigation for a quadcopter using optic flow. In AIAA Infotech@ Aerospace, page 0404. 2016.

[28] Carl John Salaan, Kenjiro Tadakuma, Yoshito Okada, Eri Takane, Kazunori Ohno, and Satoshi Tadokoro. Uav with two passive rotating hemispherical shells for physical interaction and power tethering in a complex environment. In 2017 IEEE International Conference on Robotics and Automation (ICRA), pages 3305-3312. IEEE, 2017.

[29] Carl John Salaan, Kenjiro Tadakuma, Yoshito Okada, Yusuke Sakai, Kazunori Ohno, and Satoshi Tadokoro. Development and experimental validation of aerial vehicle with passive rotating shell on each rotor. IEEE Robotics and Automation Letters, 4(3):2568-2575, 2019. 
[30] Pooya Sareh, Pisak Chermprayong, Marc Emmanuelli, Haris Nadeem, and Mirko Kovac. The spinning cyclic 'miura-oring' for mechanical collision-resilience. Origami 7, 3:981-994, 2018.

[31] Joshuah K Stolaroff, Constantine Samaras, Emma R O’Neill, Alia Lubers, Alexandra S Mitchell, and Daniel Ceperley. Energy use and life cycle greenhouse gas emissions of drones for commercial package delivery. Nature communications, 9(1):1-13, 2018.

[32] Frank Veroustraete. The rise of the drones in agriculture. EC agriculture, 2(2):325-327, 2015.

[33] Eric W Weisstein. Truncated icosahedron. https://mathworld. wolfram. com/, 2004.

[34] Jiaming Zha, Xiangyu Wu, Joseph Kroeger, Natalia Perez, and Mark W Mueller. A collisionresilient aerial vehicle with icosahedron tensegrity structure. arXiv preprint arXiv:2003.03417, 2020.

[35] Moju Zhao, Koji Kawasaki, Xiangyu Chen, Shintaro Noda, Kei Okada, and Masayuki Inaba. Whole-body aerial manipulation by transformable multirotor with two-dimensional multilinks. In 2017 IEEE International Conference on Robotics and Automation (ICRA), pages 5175-5182. IEEE, 2017. 